\title{
O Complexo Vegetacional da Zona Litorânea no Ceará: Pecém, São Gonçalo do Amarante
}

\author{
Antônio Sérgio Farias Castro ${ }^{1}$, Marcelo Freire Moro² e Marcelo Oliveira Teles de Menezes ${ }^{3,4}$
}

Recebido em 17/07/2011. Aceito em 16/01/2012

\section{RESUMO}

(O Complexo Vegetacional da Zona Litorânea no Ceará: Pecém, São Gonçalo do Amarante). O Litoral Setentrional do Nordeste (LSN) por sua localização geográfica apresenta clima mais quente e seco do que na costa leste do Brasil. Por sua proximidade com a caatinga e o cerrado, o LSN permite a co-existência de espécies destes Domínios conjuntamente com espécies de restinga, em diferentes formações, constituindo um Complexo Vegetacional. Apesar da grande importância ecológica e botânica deste ecótono, existem poucos estudos sobre a flora regional. O objetivo deste trabalho foi aprofundar o conhecimento sobre a composição florística e fitossociológica da região. Para isso, fizemos um levantamento florístico na área (entre 2007-2011), bem como consultas a registros de herbário na região e um levantamento fitossociológico em um trecho da floresta estacional semidecídua costeira (mata de tabuleiro). Foram inventariadas 382 espécies vegetais, pertencentes a 96 famílias. Na parcela fitossociológica ( 0,32 ha) foram registrados 2.970 indivíduos de 52 espécies, sendo as mais abundantes as arbóreas Manilkara triflora, Chamaecrista ensiformis e Guapira nitida e as arbustivas Cordiera sessilis e Maytenus erythroxyla (altura média 3,8 m, diâmetro médio 6,2 cm, área basal 39,28 $\mathrm{m}^{2} / \mathrm{ha}$ ). A flora local inclui elementos florísticos de caatinga, cerrado e restinga, sugerindo que a comunidade vegetal na região costeira do Ceará possui natureza ecotonal.

Palavras-chave: restinga, litoral, região costeira, vegetação, ecótono

\begin{abstract}
(The vegetation complex of the coastal zone of Ceará: Pecém, São Gonçalo do Amarante). Due to its geographical location, the northeastern Coast of Brazil (Litoral Setentrional do Nordeste - LSN) is a hotter and drier climate than the eastern coast. In addition, because of its proximity to caatinga and cerrado, the LSN contains species from these vegetation biomes and from the restinga on the coast, which comprise different plant formations and creates a vegetation complex. Despite the great importance of this ecotone, there are few studies about its flora. The objective of this work was to contribute to what is known about the floristic and phytosociological composition of this region. We made a floristic survey in the area (between 2007 and 2011), consulted herbaria data from the region and made a phytosociological study in a stretch of coastal semideciduous forest (mata de tabuleiro). The study recorded 382 plant species from 96 families. In the phytosociological survey ( $0.32 \mathrm{ha})$ we recorded 2,970 individuals and 52 species. The most abundant plants surveyed were the trees Manilkara triflora, Chamaecrista ensiformis and Guapira nitida and the shrubs Cordiera sessilis and Maytenus erythroxyla (average height $3.8 \mathrm{~m}$, average diameter $6.2 \mathrm{~cm}$, basal area $39.28 \mathrm{~m}^{2} / \mathrm{ha}$ ). The local flora includes floristic elements of caatinga, cerrado and restinga, corroborating the idea that the plant community of the coastal region of Ceará has an ecotonal nature.
\end{abstract}

Key words: restinga, littoral, coast, vegetation, ecotone

1 Pesquisador independente, Fortaleza, CE, Brasil

2 Universidade Estadual de Campinas, Instituto de Biologia, Departamento de Biologia Vegetal, Campinas, SP, Brasil

3 Instituto Federal de Educação, Ciência e Tecnologia do Ceará, Sobral, CE, Brasil

4 Autor para correspondência: mteles84@gmail.com 


\section{Introdução}

A classificação do território brasileiro em amplos domínios geográficos (e.g. Ab’Sáber 2003; Brasil 2004) inevitavelmente leva a um certo nível de generalização e imprecisão. Mapas e classificações vegetacionais de escala continental tendem a subestimar particularidades regionais na fisionomia, estrutura e composição das comunidades vegetais. Esse é o caso dos mapas e sistemas brasileiros de classificação vegetacional (e.g. Veloso et al. 1991; Brasil (2004), que muitas vezes ignoram as restingas e formações litorâneas, as quais apesar de serem faixas vegetacionais relativamente estreitas, podem atingir dezenas de quilômetros adentro do continente (Fernandes 1998). No sistema universal de classificação das vegetações brasileiras, Veloso et al. (1991), devido à escala utilizada, ignoram as peculiaridades vegetacionais de cada região, tratando toda a "vegetação com influência marinha" brasileira como uma só unidade fitogeográfica. Em seu sistema fitogeográfico, Fernandes (1998) considera as formações litorâneas de todo o Brasil como pertencentes à Sub-Província Litorânea incluída na Província Atlântica; e Brasil (2004) inclui as restingas dentro dos domínios fitogeográficos adjacentes (Fig. 1). No entanto, devido à sua extensão continental, a região litorânea do Brasil abrange uma gama de condições climáticas, geomorfológicas e pedológicas, que abrigam diferentes formações vegetais.

Enquanto a costa leste do Brasil é dominada por climas tropicais úmidos, típicos do domínio da Mata Attântica, a porção setentrional do litoral nordestino (entre o Maranhão e a chamada "curva do continente sul-americano", no Rio Grande do Norte) possui regimes climáticos bem mais secos, variando de subúmidos a semiáridos (Brasil 2002; Nimer, 1972; Ab’Sáber 2001; 2006). Essa diferença levou Ab’Saber $(2001 ; 2006)$ a individualizar esse trecho da costa como uma unidade chamada "Litoral Setentrional do Nordeste" - LSN (Fig. 1). Devido à proximidade geográfica do LSN com o domínio das Caatingas, do Cerrado e da Mata Atlântica, o LSN permite a formação de um ecótono muito peculiar: um complexo florístico-vegetacional que inclui espécies de caatinga, de cerrado, atlânticas e até amazônicas, além de outras espécies próprias das restingas (Fernandes 1990; 1998; Figueiredo 1997; Matias \& Nunes 2001; Moro et al. 2011).

Segundo Figueiredo (1997) e Fernandes (1990), na região litorânea do Ceará ocorre um grupo heterogêneo de fitofisionomias que variam desde a vegetação herbácea de pós-praia, passando por matas dunares, manchas de vegetação savânica (cerrados costeiros) até florestas estacionais semidecíduas chamadas localmente de "matas de tabuleiro", que se distinguem conspicuamente da Caatinga. No entanto, a escassez de publicações florísticas e fitossociológicas no LSN dificulta uma análise mais acurada que permita a classificação das vegetações litorâneas que ocorrem desde o Piauí até o norte do Rio Grande do Norte. Assim, visando ampliar o conhecimento sobre a flora do LSN, realizou-se um extenso levantamento florístico na região litorânea do Pecém (CE), complementando o estudo com alguns dados de estrutura da "mata de tabuleiro".

\section{Materiais e métodos}

\section{Área de Estudo}

O estudo foi realizado no distrito de Pecém, no município de São Gonçalo do Amarante, que faz parte da Região Metropolitana de Fortaleza, região litorânea do Ceará. O distrito possui $112,01 \mathrm{~km}^{2}$ e localiza-se entre $3^{\circ} 31^{\prime} 30^{\prime \prime}$ \& $3^{\circ} 41^{\prime} 20^{\prime \prime}$ S e $38^{\circ} 48^{\prime} 11^{\prime \prime} \& 38^{\circ} 56^{\prime} 14^{\prime \prime}$ O (Fig. 1; 2). Sua escolha como área de estudo deveu-se à sua heterogeneidade ambiental e à sua relevância ecológica para a conservação - alvo de instalação de um grande complexo portuário-industrial, o que tem contribuído fortemente para a supressão da vegetação em grande parte de sua área, com a consequente perda de diversidade biológica.

A região metropolitana de Fortaleza, onde a área estudada se localiza, está em uma região de transição entre regimes climáticos semiáridos e subúmidos (Nimer, 1972). A pluviosidade média em São Gonçalo do Amarante é de $1.026,4 \mathrm{~mm}$ com temperatura média anual de $26^{\circ} \mathrm{C}$, chuvas concentradas de janeiro a junho, e potencial de evapotranspiração superior à precipitação (Nimer, 1972; Ceará, 2010). Como nas demais regiões da costa cearense, o Pecém possui pouca variação altitudinal $(0-70 \mathrm{~m})$ e está situada sobre terrenos sedimentares de origem tércio-quaternária pertencentes à Formação Barreiras, além de depósitos holocênicos de areias quartzosas que formam campos de dunas e restingas de origem ainda mais recente (Campos et al. 2003; Ab'Saber 2006). Segundo Campos et al. (2003), o distrito do Pecém possui três unidades geoambientais: sertões circundantes (de origem cristalina, não estudados neste trabalho), tabuleiros pré-litorâneos (arenosos e argilo-arenosos) e planície litorânea, que inclui as praias, os campos de dunas móveis, dunas fixas, paleodunas e planícies ribeirinhas (Fig. 2). Esse estudo se restringiu às unidades tabuleiros e planície litorânea.

\section{Levantamento florístico e fitossociológico}

O levantamento florístico, que levou em consideração tanto plantas terrestres quanto aquáticas, foi realizado em duas etapas. A primeira consistiu em coletas feitas pelos autores no distrito do Pecém e em áreas muito próximas, como localidades, distritos ou municípios vizinhos: localidades de Paú, Varjota, Parada, Jacarecoara e Siupé, em São Gonçalo do Amarante; e Maceió, Praíba, Lagoa Amarela e Matões, em Caucaia (Fig. 2). As coletas foram realizadas pelo método de caminhamento (Filgueiras et al. 1994) entre os anos de 2007 e 2011, em pontos amostrais distribuídos entre a beira da praia (ao norte) até a planície de inundação da Lagoa do Gereraú (ponto de coleta mais ao sul). A florística também incluiu espécies exóticas que tivessem atingido a categoria 


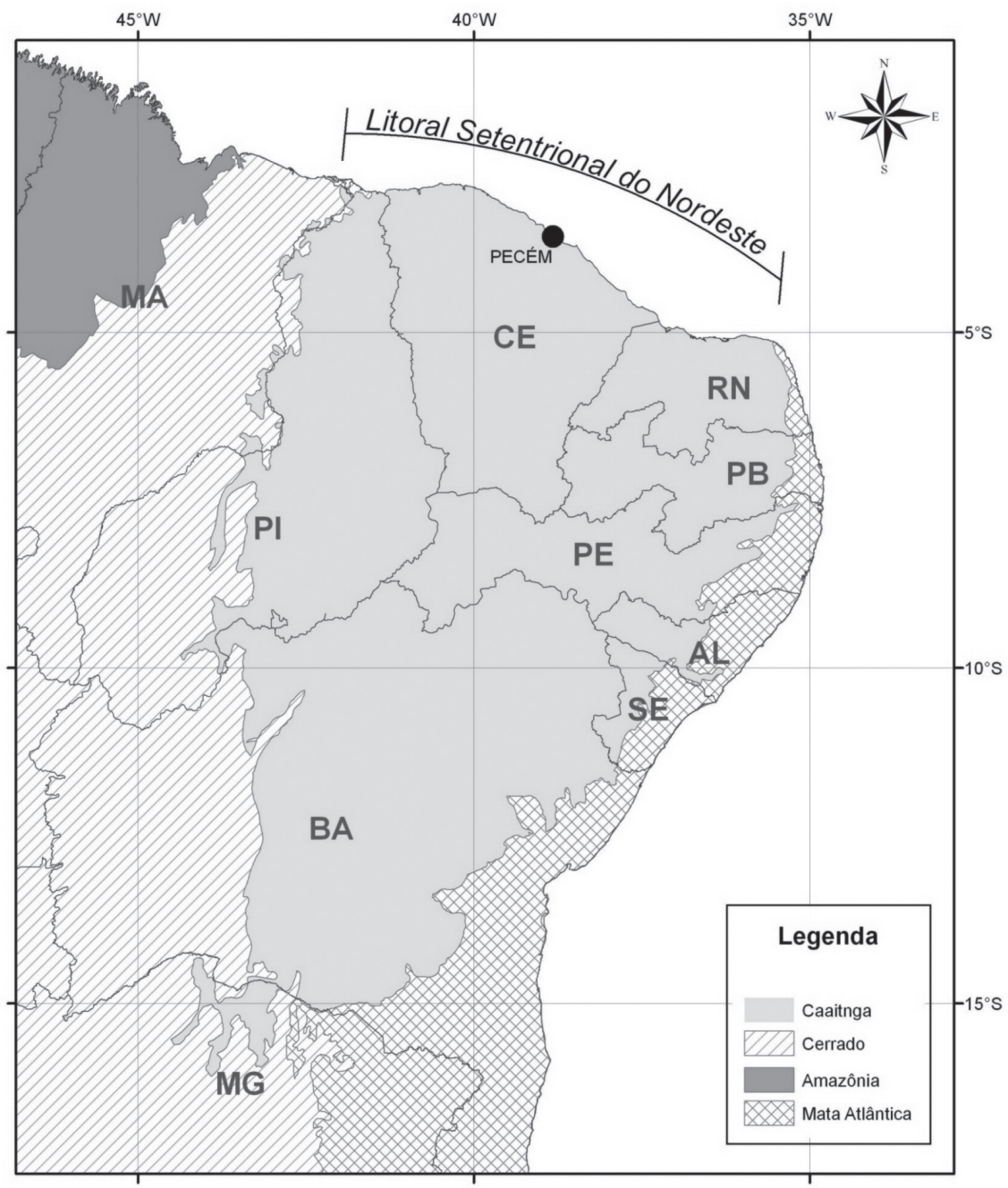

Figura 1. Localização da área de estudo (Pecém) no "Litoral Setentrional do Nordeste" (Ab’Sáber, 2001), região costeira classificada por Brasil (2004) dentro do domínio das Caatingas. Adaptado de Brasil (2004).

de invasora no local (sensu Richardson et al. 2000). A exótica Acacia mangium (EAC 47.401), cultivada próximo à Estação Ecológica do Pecém, por exemplo, ainda não havia atingido esse estágio e foi excluída da lista, embora seja invasora em outros locais.

Todos os espécimes coletados foram tombados no herbário EAC, da Universidade Federal do Ceará. O levantamento foi complementado com a listagem de todas as exsicatas do Pecém depositadas no herbário até o primeiro semestre de 2011. Todos os registros foram conferidos e tiveram suas identificações atualizadas ou corrigidas.

Embora o distrito do Pecém inclua áreas de caatinga ao sul, este estudo se limitou ao complexo vegetacional litorâneo, ou seja, fitofisionomias localizadas sobre tabuleiros pré-litorâneos da Formação Barreiras, lagoas litorâneas, dunas fixas, semi-fixas e móveis, vegetação de pós-praia, várzeas de rios e lagoas (carnaubais), e manguezais. Terrenos sobre o embasamento cristalino - ao sul da planície de inundação da Lagoa do Gereraú (Fig. 2) - não foram incluídos nas coletas. Para registrar em que ambientes as espécies ocorrem, foram utilizadas as categorias de ambientes litorâneos do sistema de unidades fitoecológicas do Ceará (Figueiredo, 1997), com adaptações:

Vegetação pioneira psamófila I - vegetação que ocorre no pós-praia, sujeita à influência marinha e ao excesso de sal;

Vegetação pioneira psamófila II - vegetação que ocorre sobre as dunas móveis e semi-fixas, sob regime de elevada motilidade dos sedimentos arenosos e extrema radiação solar;

Floresta de dunas fixas e retaguarda de dunas - vegetação dominada por espécies de porte arbóreo-arbustivo, associadas ao campo de dunas fixas edafizadas;

Vegetação dos tabuleiros pré-litorâneos - localizada sobre os terrenos da Formação Barreiras. Sua fisionomia pode variar de floresta semidecídua (mata de tabuleiro) a savanas costeiras (cerrados costeiros);

Vegetação aquática e paludosa de lagoas e brejos vegetação de baixios, lagoas e rios de fluxo lento, tanto à retaguarda das dunas como nos tabuleiros; 


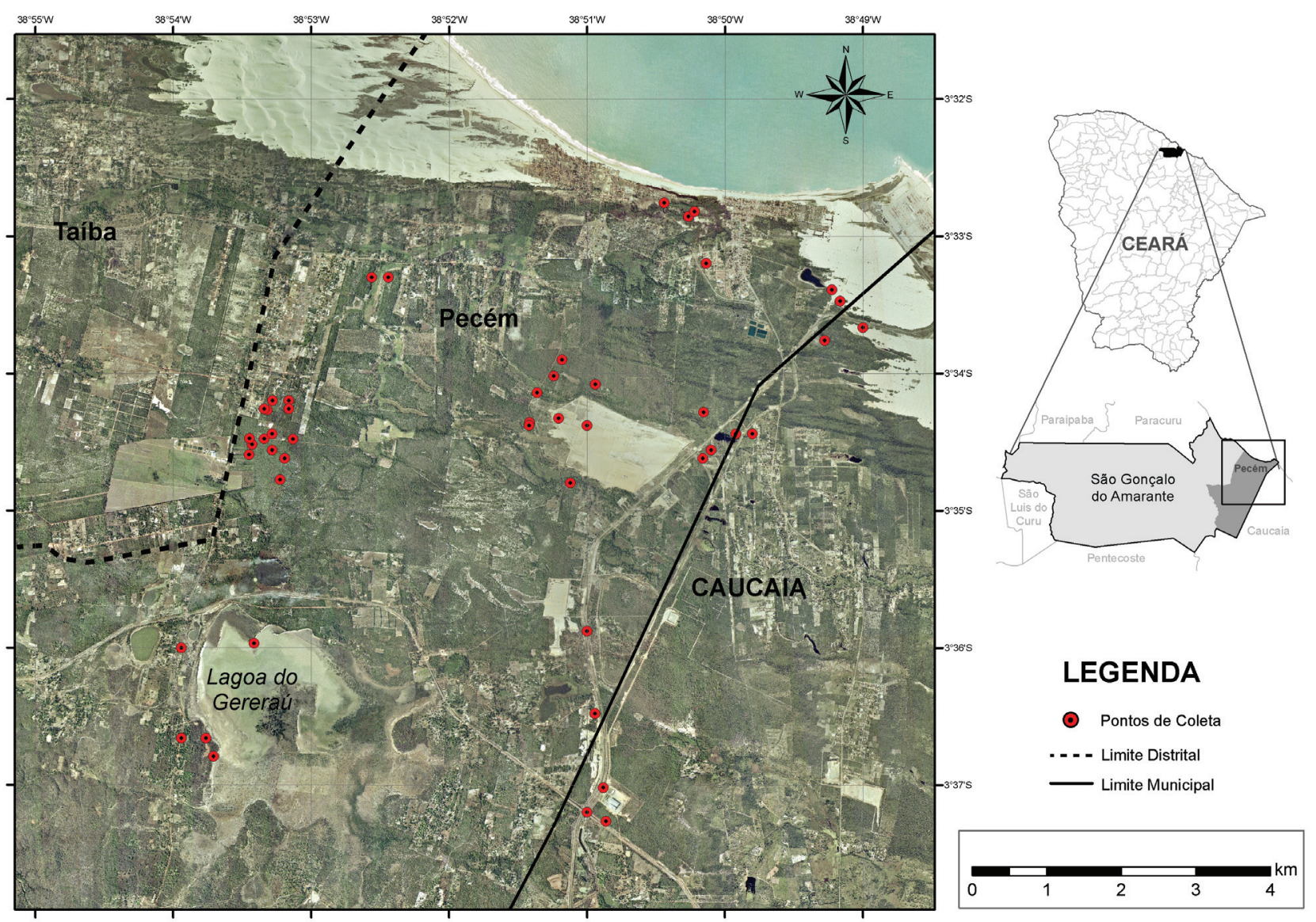

Figura 2. Localização dos principais pontos de amostragem florística no distrito de Pecém (São Gonçalo do Amarante, Ceará). Elaboração: M.O.T. Menezes

Floresta mista dicótilo-palmácea (Carnaubais) - vegetação de várzeas com presença conspícua da carnaúba (Copernicia prunifera), especialmente no entorno da Lagoa do Gereraú. Também pode ocorrer em áreas mais continentais, onde é considerada um subtipo de caatinga (Andrade-Lima 1981);

Manguezal - vegetação florestal paludosa, halófila, típica de regiões estuarinas; inclui ecossistemas campestres associados - apicuns e salgados.

Para o levantamento fitossociológico da mata de tabuleiro, foi selecionado um trecho de mata secundária em estado razoável de conservação e foram estabelecidas 8 parcelas de $20 \times 20 \mathrm{~m}$, totalizando $0,32 \mathrm{ha}$, em áreas no entorno do Jardim Botânico de São Gonçalo do Amarante (coordenada de referência: $3^{\circ} 34^{\prime} 35^{\prime \prime} S \& 38^{\circ} 53^{\prime} 02^{\prime \prime}$ ) ). A floresta selecionada aparentemente não sofre corte raso há muitos anos, mas o corte seletivo de madeira (especialmente Manilkara triflora e Chamaecrista ensiformis) era evidente. O levantamento foi realizado de acordo com a metodologia proposta por Durigan (2003). Todos os indivíduos lenhosos (incluindo cipós) com Diâmetro ao Nível do Solo (DNS) maior ou igual a $3 \mathrm{~cm}$ foram incluídos na amostragem. Registrou-se para cada indivíduo a espécie, o DNS (medido com suta den- drométrica) e a altura da planta. O sistema de classificação taxonômica adotado foi APG III (2009).

\section{Resultados e discussão}

O levantamento florístico registrou 382 espécies pertencentes a 96 famílias. A família mais rica foi Fabaceae com 69 espécies. Outras famílias ricas em espécies foram Cyperaceae (22 espécies), Rubiaceae (20 espécies), Poaceae (17), Euphorbiaceae (15), Myrtaceae (15), Malvaceae (14) e Bignoniaceae (13), as quais somaram $48 \%$ da flora amostrada (Tab. 1).

$\mathrm{Na}$ fitossociologia foram amostrados 2.970 indivíduos vivos (52 espécies, 29 famílias) e 118 mortos (ainda de pé) nos 0,32 ha (Índice de Shannon $=2,8$ nats/indivíduos). A densidade foi de 9.281,25 ind/ha. As espécies arbóreas Manilkara triflora, Chamaecrista ensiformis e Guapira nitida e as arbustivas Cordiera sessilis e Maytenus erythroxyla foram as mais abundantes no local, totalizando 1.747 plantas (58,8\% dos indivíduos - Tabela 2).

O diâmetro médio da comunidade foi de $6,2 \mathrm{~cm}$ e desvio padrão de 3,90 cm, com $81 \%$ dos indivíduos com diâmetros de até $8 \mathrm{~cm}$ (Fig. 3). A altura média da comunidade (considerando apenas árvores e arbustos e excluindo cipós) foi 
Tabela 1. Lista florística da região litorânea do Pecém, Ceará. Hábitos: Arv - árvore; Arb - arbusto; Subarb - subarbusto; Arb-es - arbusto escandente; Her - erva; Trep - trepadeira; Hemi-pa - hemi-parasita; Paras - parasita; Epif - epífita. Unidades Fitoecológicas: P1 - Vegetação pioneira psamófila I (pós-praia); P2 - Vegetação pioneira psamófila II (dunas móveis e semi-fixas); D - Floresta de dunas fixas e de retaguarda de dunas; T - Vegetação dos tabuleiros pré-litorâneos; A - Vegetação aquática e paludosa de lagoas e brejos; C - Floresta mista dicótilo-palmácea (carnaubal); M - Manguezal. Ocorrência: X - Ocorrência da espécie em dada unidade fitoecológica; d - Ocorrência da espécie em dada unidade fitoecológica registrada apenas em ambientes altamente perturbados (áreas desmatadas e beiras de estradas). ${ }^{*}$ ) Espécies cuja presença no Pecém foi confirmada pelos autores, mas cujas coletas depositadas no herbário foram feitas em áreas circunvizinhas à área de estudo. Invasora: Espécie exótica invasora para a área estudada (sensu Richardson et al. 2000).

\begin{tabular}{|c|c|c|c|c|c|c|c|c|c|c|c|c|}
\hline Espécie & EAC & Coletor & $\mathrm{NC}$ & Nome popular & Hábito & P1 & $\mathrm{P} 2$ & $\mathrm{D}$ & $\mathrm{T}$ & A & $\mathrm{C}$ & M \\
\hline \multicolumn{13}{|c|}{ Pteridófitas } \\
\hline \multicolumn{13}{|l|}{ PTERIDACEAE } \\
\hline Acrostichum aureum $\mathrm{L}$. & 29205 & Lopes, M. S. & sn & Samambaia & Arb & & & & & $\mathrm{X}$ & & \\
\hline Ceratopteris pteridoides (Hook.) Hieron. & 29206 & Lopes. M. S. & sn & & Her & & & & & $\mathrm{X}$ & & \\
\hline \multicolumn{13}{|l|}{ SALVINIACEAE } \\
\hline Salvinia auriculata Aubl. & 41536 & Moro, M. F. & 344 & & Her & & & & & $\mathrm{X}$ & & \\
\hline \multicolumn{13}{|l|}{ SCHIZAEACEAE } \\
\hline Actinostachys pennula (Sw.) Hook. & 44942 & Moro, M. F. & 709 & & Her & & & & $\mathrm{X}$ & $\mathrm{X}$ & & \\
\hline \multicolumn{13}{|l|}{ THELYPTERIDACEAE } \\
\hline Thelypteris interrupta (Willd.) K.Iwats. & 48158 & Castro, A. S. F. & 2385 & Samambaia & Her & & & & $\mathrm{X}$ & & & \\
\hline \multicolumn{13}{|c|}{ Angiospermas } \\
\hline \multicolumn{13}{|l|}{ ACANTHACEAE } \\
\hline Avicennia germinans (L.) L. & 42569 & Moro, M. F. & 349 & Mangue-preto & Arv & & & & & & & $\mathrm{X}$ \\
\hline Justicia aequilabris (Nees) Lindau & 47389 & Castro, A. S. F. & 2330 & & SubArb & & & & $\mathrm{X}$ & & & \\
\hline Ruellia asperula (Mart. ex Nees) Lindau & 50178 & Magalhães, H. & 228 & Melosa & SubArb & & & $\mathrm{X}$ & $\mathrm{X}$ & $\mathrm{X}$ & & \\
\hline Ruellia bahiensis (Nees) Morong & 47405 & Castro, A. S. F. & 2346 & & Her & & & & $\mathrm{X}$ & & & \\
\hline \multicolumn{13}{|l|}{ AIZOACEAE } \\
\hline Sesuvium portulacastrum (L.) L. & 42564 & Moro, M. F. & 354 & Beldroega & Her & $\mathrm{X}$ & & & & & & $\mathrm{X}$ \\
\hline \multicolumn{13}{|l|}{ ALISMATACEAE } \\
\hline Echinodorus subalatus (Mart.) Griseb. & 41553 & Moro, M. F. & 281 & & Her & & & & & $\mathrm{X}$ & & \\
\hline Hydrocleys nymphoides (Willd.) Buchenau & 42545 & Moro, M. F. & 285 & & Her & & & & & $\mathrm{X}$ & & \\
\hline \multicolumn{13}{|l|}{ AMARANTHACEAE } \\
\hline Alternanthera brasiliana (L.) Kuntze & 50171 & Magalhães, H. & 231 & & SubArb & & & $\mathrm{X}$ & $\mathrm{X}$ & $\mathrm{X}$ & & \\
\hline Blutaparon portulacoides (A.St.-Hil.) Mears & 42575 & Moro, M. F. & 352 & Pirrixio & Her & $\mathrm{X}$ & & & & & & $\mathrm{X}$ \\
\hline \multicolumn{13}{|l|}{ ANACARDIACEAE } \\
\hline Anacardium occidentale L. & 37779 & Roberto, I. J. & 5 & Cajueiro & Arv & & $\mathrm{X}$ & $\mathrm{X}$ & $\mathrm{X}$ & & & \\
\hline Tapirira guianensis Aubl. & 41526 & Moro, M. F. & 321 & Pau-pombo & Arv & & & & $\mathrm{X}$ & & & \\
\hline \multicolumn{13}{|l|}{ ANNONACEAE } \\
\hline Annona coriacea Mart. & 41563 & Menezes, M. O. T. & 11 & Araticum & Arv & & & & $\mathrm{X}$ & & & \\
\hline Duguetia riedeliana R.E.Fr. & 47769 & Castro, A. S. F. & 2354 & Mium & Arv & & & & $\mathrm{X}$ & & & \\
\hline Xylopia sericea A.St.-Hil. & 44648 & Ferreira, R. G. & 59 & Embiriba & Arv & & & & $\mathrm{X}$ & & & \\
\hline \multicolumn{13}{|l|}{ APOCYNACEAE } \\
\hline Allamanda blanchetii A.DC. & 47768 & Castro, A. S. F. & 2366 & Quatro-patacas & Arb & & & & $\mathrm{X}$ & & $\mathrm{X}$ & \\
\hline Aspidosperma ulei Markgr. & 48164 & Castro, A. S. F. & 2391 & Piquiá & Arv & & & $\mathrm{X}$ & $\mathrm{X}$ & & & \\
\hline Calotropis procera (Aiton) W.T. Aiton (invasora) & 28218 & Sampaio, D. S. & 23 & Hortênsia & Arb & & $\mathrm{X}$ & & $\mathrm{X}$ & & $\mathrm{X}$ & \\
\hline Cryptostegia madagascariensis Bojer (invasora) & 44950 & Sobrinho, M. S. & sn & Viúva-alegre & Arb-Es & & & & & & $\mathrm{X}$ & \\
\hline Cynanchum roulinioides (E. Fourn.) Rapini & 49166 & Castro, A. S. F. & 2498 & & Trep & & & & $\mathrm{X}$ & & & \\
\hline Hancornia speciosa Gomes & 46024 & Ferreira, R. G. & 114 & Mangaba & Arv & & & & $\mathrm{X}$ & & & \\
\hline Himatanthus drasticus (Mart.) Plumel & 41493 & Moro, M. F. & 248 & Janaguba & Arv & & & & $\mathrm{X}$ & & & \\
\hline $\begin{array}{l}\text { Mandevilla scabra (Hoffmanns. ex Roem. \& } \\
\text { Schult.) K.Schum. }\end{array}$ & 42567 & Moro, M. F. & 373 & & Trep & & & & $\mathrm{X}$ & & $\mathrm{X}$ & \\
\hline Matelea ganglinosa (Vell.) Rapini * & 38454 & Castro, A. S. F. & 1608 & & Trep & & & & $\mathrm{X}$ & & & \\
\hline Tabernaemontana catharinensis A.DC. & 41499 & Moro, M. F. & 235 & Grão-de-galo & Arb & & & & $\mathrm{X}$ & & & \\
\hline \multicolumn{13}{|l|}{ ARACEAE } \\
\hline Montrichardia linifera (Arruda) Schott & 44924 & Moro, M. F. & 397 & Aninga & Her & & & & & $\mathrm{X}$ & & \\
\hline Philodendron acutatum Schott & 47406 & Castro, A. S. F. & 2347 & Cipó-de-fonte & Trep & & & & $\mathrm{X}$ & $\mathrm{X}$ & $\mathrm{X}$ & \\
\hline Pistia stratiotes $\mathrm{L}$. & 47410 & Castro, A. S. F. & 2351 & Pasta & Her & & & & & $\mathrm{X}$ & & \\
\hline Taccarum ulei (Engl.) \& K. Krause * & 49113 & Castro, A. S. F. & 2480 & Milho-de-cobra & Her & & & $\mathrm{X}$ & $\mathrm{X}$ & & & \\
\hline \multicolumn{13}{|l|}{ ARALIACEAE } \\
\hline Hydrocotyle bonariensis Lam. * & 49112 & Castro, A. S. F. & 2479 & Vintém & Her & & & & & $\mathrm{X}$ & & \\
\hline \multicolumn{13}{|l|}{ ARECACEAE } \\
\hline Copernicia prunifera (Mill.) H.E.Moore & 47803 & Castro, A. S. F. & 2363 & Carnaúba & Arv & & & & & & $\mathrm{X}$ & \\
\hline ARISTOLOCHIACEAE & & & & & & & & & & & & \\
\hline
\end{tabular}


Tabela 1. Continuação

\begin{tabular}{|c|c|c|c|c|c|c|c|c|c|c|c|}
\hline Espécie & EAC & Coletor & $\mathrm{NC}$ & Nome popular & Hábito & P1 & $\mathrm{P} 2$ & $\mathrm{D}$ & $\mathrm{T}$ & A & $\mathrm{C} \mathrm{M}$ \\
\hline Aristolochia birostris Duch. & 50346 & Castro, A. S. F. & 2584 & Jericó & Trep & & & & $\mathrm{X}$ & & \\
\hline \multicolumn{12}{|l|}{ ASTERACEAE } \\
\hline Bidens bipinnata L. (invasora) & 50140 & Magalhães, H. & 201 & & SubArb & & & $\mathrm{X}$ & $\mathrm{X}$ & & \\
\hline Blainvillea acmella (L.) Philipson & 49125 & Castro, A. S. F. & 2482 & & Arb & & & & $\mathrm{X}$ & & \\
\hline Egletes viscosa (L.) Less. & 24475 & Silveira, E. & sn & Macela & Her & & & & $\mathrm{X}$ & $\mathrm{X}$ & \\
\hline Elephantopus hirtiflorus DC. & 41512 & Moro, M. F. & 295 & Língua-de-vaca & Her & & $\mathrm{X}$ & $\mathrm{X}$ & $\mathrm{X}$ & $\mathrm{X}$ & \\
\hline Emilia sonchifolia (L.) DC. ex Wight & 50153 & Magalhães, H. & 9 & & Her & & & $\mathrm{X}$ & $\mathrm{X}$ & & \\
\hline Lepdaploa remotiflora (Rich.) H. Rob. & 50164 & Magalhães, H. & 238 & & SubArb & & & & $\mathrm{X}$ & & \\
\hline Melampodium divaricatum (Rich. ex Pers.) DC. & 49027 & Castro, A. S. F. & 2452 & & Her & & & & $\mathrm{X}$ & & \\
\hline Mikania cordifolia (L.f.) Willd. & 47394 & Castro, A. S. F. & 2335 & & Trep & & & $\mathrm{X}$ & $\mathrm{X}$ & $\mathrm{X}$ & \\
\hline Porophyllum ruderale (Jacq.) Cass. & 49129 & Castro, A. S. F. & 2486 & Cravo-de-urubu & Her & & $\mathrm{X}$ & & $\mathrm{X}$ & & \\
\hline Sphagneticola trilobata (L.) Pruski & 50168 & Magalhães, H. & 240 & & Her & & & $\mathrm{X}$ & $\mathrm{X}$ & & \\
\hline Stilpnopappus trichospiroides Mart. ex DC. & 49237 & Castro, A. S. F. & 2500 & & Her & & $\mathrm{X}$ & & $\mathrm{x}$ & & \\
\hline Tilesia baccata (L.f.) Pruski (invasora) & 44923 & Moro, M. F. & 396 & & Arb & & & $\mathrm{X}$ & $\mathrm{x}$ & & \\
\hline Wedelia villosa Gardner & 47397 & Castro, A. S. F. & 2338 & Camará & Arb & & & & $\mathrm{x}$ & $\mathrm{X}$ & \\
\hline \multicolumn{12}{|l|}{ BIGNONIACEAE } \\
\hline $\begin{array}{l}\text { Adenocalymma pedunculatum (Vell.) } \\
\text { L.G.Lohmann }\end{array}$ & 41583 & Moro, M. F. & 270 & Canga-de-boi & Arb-es & & & & $\mathrm{x}$ & & \\
\hline Bignonia binata Thunb. * & 31373 & Castro, A. S. F. & 1167 & & Arb-es & & & & $\mathrm{x}$ & & $\mathrm{X}$ \\
\hline Cuspidaria argentea (Wawra) Sandwith & 31379 & Castro, A. S. F. & 1173 & & Arb-es & & & & $\mathrm{x}$ & & \\
\hline Dolichandra unguis-cati (L.) L.G. Lohmann & 50145 & Magalhães, H. & 212 & & Trep & & & $\mathrm{X}$ & $\mathrm{x}$ & & \\
\hline $\begin{array}{l}\text { Fridericia dispar (Bureau \& K. Schum.) L.G. } \\
\text { Lohmann * }\end{array}$ & 48028 & Castro, A. S. F. & 2379 & & Trep & & & & $\mathrm{x}$ & & \\
\hline $\begin{array}{l}\text { Fridericia subverticillata (Bureau \& K.Schum.) } \\
\text { L.G.Lohmann }\end{array}$ & 47774 & Castro, A. S. F. & 2367 & & Trep & & & & $\mathrm{x}$ & & \\
\hline Handroanthus impetiginosus Mattos & 48171 & Castro, A. S. F. & 2398 & Pau-d’arco-roxo & Arv & & & $\mathrm{X}$ & $\mathrm{x}$ & & \\
\hline Jacaranda sp. & 48852 & Ferreira, R. G. & sn & & Arv & & & & $\mathrm{x}$ & & \\
\hline Lundia cordata (Vell.) DC. & 48021 & Castro, A. S. F. & 2372 & & Trep & & & $\mathrm{X}$ & $\mathrm{x}$ & & \\
\hline Mansoa sp. & 41560 & Menezes, M. O. T. & 15 & & Trep & & & & $\mathrm{x}$ & & \\
\hline $\begin{array}{l}\text { Neojobertia candolleana (Mart. ex DC.) Bureau \& } \\
\text { K.Schum. }\end{array}$ & 48020 & Castro, A. S. F. & 2370 & & Trep & & & & $\mathrm{x}$ & & \\
\hline $\begin{array}{l}\text { Tabebuia aurea (Silva Manso) Benth. \& Hook.f. ex } \\
\text { S.Moore }\end{array}$ & 44665 & Ferreira, R. G. & 77 & Caraúba & Arv & & & & $\mathrm{x}$ & & $\mathrm{x}$ \\
\hline Tabebuia roseoalba (Ridl.) Sandwith & 48825 & Moro, M. F. & 720 & Peroba & Arv & & & $\mathrm{x}$ & $\mathrm{x}$ & & \\
\hline \multicolumn{12}{|l|}{ BIXACEAE } \\
\hline \multicolumn{12}{|l|}{ BORAGINACEAE } \\
\hline Cordia rufescens A.DC. & 31376 & Castro, A. S. F. & 1170 & Grão-de-galo & Arv & & & & $\mathrm{X}$ & & \\
\hline Euploca polyphylla (Lehm.) J.I.M.Melo \& Semir & 25024 & Oliveira, E. R. & sn & & Her & & $\mathrm{X}$ & & $\mathrm{X}$ & $\mathrm{X}$ & \\
\hline Tournefortia candidula (Miers) Johnst. & 41578 & Menezes, M. O. T. & 26 & & Trep & & & $\mathrm{x}$ & $\mathrm{x}$ & & \\
\hline Varronia leucomalloides (Taroda) J. S. Mill. * & 39672 & Castro, A. S. F. & 1759 & & Arb & & & $\mathrm{x}$ & $\mathrm{x}$ & & \\
\hline \multicolumn{12}{|l|}{ BROMELIACEAE } \\
\hline Bromelia karatas $\mathrm{L}$. & 49003 & Castro, A. S. F. & 2353 & Croatá & Her & & & $\mathrm{x}$ & $\mathrm{x}$ & & $\mathrm{X}$ \\
\hline \multicolumn{12}{|l|}{ BURSERACEAE } \\
\hline Commiphora leptophloeos (Mart.) J.B.Gillett & 48912 & Ferreira, R. G. & sn & Imburana & Arv & & & $\mathrm{X}$ & $\mathrm{X}$ & & \\
\hline Protium heptaphyllum (Aubl.) Marchand & 41497 & Moro, M. F. & 241 & Almescla & Arv & & & $\mathrm{x}$ & $\mathrm{X}$ & & \\
\hline \multicolumn{12}{|l|}{ CACTACEAE } \\
\hline Cereus jamacaru DC. & 47801 & Castro, A. S. F. & 2365 & Mandacaru & Arv & & & $\mathrm{x}$ & $\mathrm{X}$ & & $\mathrm{X}$ \\
\hline $\begin{array}{l}\text { Pilosocereus catingicola subsp. salvadorensis } \\
\text { (Werderm.) Zappi }\end{array}$ & 47770 & Castro, A. S. F. & 2355 & Cardeiro & Arv & & $\mathrm{X}$ & $\mathrm{x}$ & $\mathrm{X}$ & & $\mathrm{X}$ \\
\hline \multicolumn{12}{|l|}{ CANNABACEAE } \\
\hline Trema micrantha (L.) Blume & 47409 & Castro, A. S. F. & 2350 & Piriquiteira & Arv & & & $\mathrm{x}$ & $\mathrm{X}$ & & \\
\hline \multicolumn{12}{|l|}{ CAPPARACEAE } \\
\hline Crateva tapia $\mathrm{L}$. & 48561 & Castro, A. S. F. & 2418 & Trapiá & Arv & & & $\mathrm{x}$ & $\mathrm{X}$ & & $\mathrm{X}$ \\
\hline Cynophalla hastata (Jacq.) J.Presl & 33079 & Lima, M. F. & sn & Feijão-bravo & Arv & & & $\mathrm{x}$ & $\mathrm{X}$ & & \\
\hline \multicolumn{12}{|l|}{ CELASTRACEAE } \\
\hline Maytenus erythroxyla Reissek & 46035 & Ferreira, R. G. & 118 & Casca-grossa & Arv-Arb & & & $\mathrm{x}$ & $\mathrm{X}$ & & \\
\hline Maytenus obtusifolia Mart. * & 44584 & Castro, A. S. F. & 2152 & Casca-grossa & Arv & & & & & & $\mathrm{X}$ \\
\hline
\end{tabular}


Tabela 1. Continuação

\begin{tabular}{|c|c|c|c|c|c|c|c|c|c|c|c|c|}
\hline Espécie & EAC & Coletor & $\mathrm{NC}$ & Nome popular & Hábito & P1 & $\mathrm{P} 2$ & $\mathrm{D}$ & $\mathrm{T}$ & A & $\mathrm{C} \mathrm{M}$ & M \\
\hline \multicolumn{13}{|l|}{ CHRYSOBALANACEAE } \\
\hline Chrysobalanus icaco L. & 41467 & Moro, M. F. & 292 & Guajiru & Arb & $\mathrm{X}$ & & $\mathrm{X}$ & $\mathrm{X}$ & $\mathrm{X}$ & & \\
\hline Hirtella ciliata Mart. \& Zucc. & 41508 & Moro, M. F. & 243 & Açoita-cavalo & Arb-Arv & & & & $\mathrm{X}$ & & & \\
\hline Hirtella racemosa Lam. & 41510 & Moro, M. F. & 245 & & Arb & & & & $\mathrm{X}$ & & & \\
\hline Licania tomentosa (Benth.) Fritsch & 49080 & Castro, A. S. F. & 2468 & Goiti & Arv & & & & $\mathrm{X}$ & & & \\
\hline Parinari campestris Aubl. & 46025 & Ferreira, R. G. & 113 & Oiticica-brava & Arv & & & & $\mathrm{X}$ & & & \\
\hline \multicolumn{13}{|l|}{ CLEOMACEAE } \\
\hline Tarenaya spinosa (Jacq.) Raf. & 33748 & Vieira, A. V. & sn & Mussambê & Arb & & & & & $\mathrm{X}$ & $\mathrm{X}$ & \\
\hline \multicolumn{13}{|l|}{ CLUSIACEAE } \\
\hline Clusia panapanari (Aubl.) Choisy & 42566 & Moro, M. F. & 377 & & Arb & & & & $\mathrm{X}$ & & & \\
\hline \multicolumn{13}{|l|}{ COMBRETACEAE } \\
\hline Buchenavia tetraphylla (Aubl.) R.A.Howard & 46989 & Ferreira, R. G. & 127 & Embiridiba & Arv & & & & $\mathrm{X}$ & & & \\
\hline Combretum glaucocarpum Mart. & 48170 & Castro, A. S. F. & 2397 & Sipaúba & Arv & & & & $\mathrm{X}$ & & & \\
\hline Combretum leprosum Mart. & 49034 & Castro, A. S. F. & 2459 & Mofumbo & Arb & & & $\mathrm{X}$ & $\mathrm{X}$ & & $\mathrm{X}$ & \\
\hline Conocarpus erectus $\mathrm{L}$. & 42573 & Moro, M. F. & 345 & Mangue-de-botão & Arv & & & & & & & $\mathrm{X}$ \\
\hline Laguncularia racemosa (L.) C.F.Gaertn. & 42574 & Moro, M. F. & 346 & Mangue-de-sapateiro & Arv & & & & & & & $\mathrm{X}$ \\
\hline \multicolumn{13}{|l|}{ COMMELINACEAE } \\
\hline Commelina diffusa Burm.f. & 41480 & Moro, M. F. & 313 & & Her & & $\mathrm{X}$ & & $\mathrm{X}$ & $\mathrm{X}$ & & \\
\hline Tradescantia ambigua Mart. & 49994 & Magalhães, H. & 261 & & Her & & & $\mathrm{X}$ & & & & \\
\hline \multicolumn{13}{|l|}{ CONVOLVULACEAE } \\
\hline Evolvulus ovatus Fernald & 49086 & Castro, A. S. F. & 2472 & & Her & & $\mathrm{X}$ & & $\mathrm{X}$ & & & \\
\hline Ipomoea asarifolia (Desr.) Roem. \& Schult. & 37775 & Roberto, I. J. & 1 & Salsa & Her & $\mathrm{X}$ & $\mathrm{X}$ & & $\mathrm{X}$ & $\mathrm{X}$ & $\mathrm{X}$ & \\
\hline Ipomoea bahiensis Willd. ex Roem. \& Schult. & 49127 & Castro, A. S. F. & 2484 & & Trep & & $\mathrm{X}$ & & $\mathrm{X}$ & & & \\
\hline Ipomoea blanchetii Choisy & 49032 & Castro, A. S. F. & 2457 & & Trep & & & $\mathrm{X}$ & $\mathrm{X}$ & & & \\
\hline Ipomoea pes-caprae (L.) R.Br. & 28435 & Sampaio, D. S. & 28 & Salsa-da-praia & Her & $\mathrm{X}$ & $\mathrm{X}$ & & & & & \\
\hline Ipomoea subincana (Choisy) Meisn. & 49025 & Castro, A. S. F. & 2450 & Batata-de-porco & Trep & & & $\mathrm{X}$ & $\mathrm{X}$ & & $\mathrm{X}$ & \\
\hline Jacquemontia serrata (Choisy) Meisn. & 39574 & Castro, A. S. F. & 1708 & & Her & & $\mathrm{X}$ & & $\mathrm{X}$ & & & \\
\hline Jacquemontia tamnifolia (L.) Griseb. & 48161 & Castro, A. S. F. & 2388 & & Trep & & & & $\mathrm{X}$ & & & \\
\hline Merremia cissoides (Lam.) Hallier f. & 48173 & Castro, A. S. F. & 2400 & & Trep & & & & $\mathrm{X}$ & & & \\
\hline Operculina alata (Ham.) Urb. & 49087 & Castro, A. S. F. & 2473 & Batata-de-purga & Trep & & & & $\mathrm{X}$ & & & \\
\hline \multicolumn{13}{|l|}{ CUCURBITACEAE } \\
\hline Apodanthera congestiflora Cogn. * & 38217 & Castro, A. S. F. & 1605 & Cabeça-de-negro & Trep & & & $\mathrm{X}$ & $\mathrm{X}$ & & & \\
\hline \multicolumn{13}{|l|}{ CYPERACEAE } \\
\hline Bulbostylis scabra (J.Presl \& C.Presl) C.B.Clarke & 49031 & Castro, A. S. F. & 2456 & & Her & & $\mathrm{X}$ & & $\mathrm{X}$ & $\mathrm{X}$ & & \\
\hline Cyperus aggregatus (Willd.) Endl. & 27570 & Amado, R. & 62 & & Her & & & & & $\mathrm{X}$ & & \\
\hline Cyperus articulatus $\mathrm{L}$. & 29399 & Amado, R. & 199 & & Her & & & & & $\mathrm{X}$ & & \\
\hline Cyperus haspan $\mathrm{L}$. & 41554 & Moro, M. F. & 316 & & Her & & & & & $\mathrm{X}$ & & \\
\hline Cyperus ligularis $\mathrm{L}$. & 42543 & Moro, M. F. & 355 & Capim-açu & Her & & $\mathrm{X}$ & & $\mathrm{X}$ & $\mathrm{X}$ & $\mathrm{X}$ & \\
\hline Cyperus maritimus Poir. & 33530 & Vieira, A. V. & sn & & Her & $\mathrm{X}$ & $\mathrm{X}$ & & & & & \\
\hline Cyperus surinamensis Rottb. & 31036 & Amado, R. & 2046 & & Her & & & $\mathrm{X}$ & $\mathrm{X}$ & & & \\
\hline Eleocharis atropurpurea (Retz.) J.Presl \& C.Presl & 31041 & Amado, R. & 205 & & Her & & & & & $\mathrm{X}$ & & \\
\hline Eleocharis flavescens (Poir.) Urb. & 23435 & Castro, A. S. F. & 46 & & Her & & & & & $\mathrm{X}$ & & \\
\hline Eleocharis geniculata (L.) Roem. \& Schult. & 41555 & Moro, M. F. & 284 & & Her & & & & & $\mathrm{X}$ & & \\
\hline Eleocharis interstincta (Vahl) Roem. \& Schult. & 41528 & Moro, M. F. & 318 & Junco & Her & & & & & $\mathrm{X}$ & & \\
\hline Fimbristylis cymosa $\mathrm{R} . \mathrm{Br}$. & 27564 & Oliveira, E. R. A. & 64 & & Her & & $\mathrm{X}$ & & & $\mathrm{X}$ & & \\
\hline Fuirena umbellata Rottb. & 41531 & Moro, M. F. & 315 & & Her & & & & & $\mathrm{X}$ & & \\
\hline Kyllinga brevifolia Rottb. * & 39718 & Castro, A. S. F. & 1787 & & Her & & & & & $\mathrm{X}$ & & \\
\hline Kyllinga vaginata Lam. & 29319 & Amado, R. & 206 & & Her & & & & & $\mathrm{X}$ & & \\
\hline $\begin{array}{l}\text { Lagenocarpus verticillatus (Spreng.) T. Koyama \& } \\
\text { Maguire }\end{array}$ & 49111 & Castro, A. S. F. & 2478 & & Her & & & & $\mathrm{X}$ & & & \\
\hline Pycreus polystachyos (Rottb.) P.Beauv. & 27567 & Amado, R. & 61 & & Her & & & & & $\mathrm{X}$ & & \\
\hline Remirea maritima Aubl. & 33084 & Lima, M. F. & sn & Barba-de-bode & Her & $\mathrm{X}$ & $\mathrm{X}$ & & & & & \\
\hline Rhynchospora holoschoenoides (Rich.) Herter & 37782 & Roberto, I. J. & sn & & Her & & & & & $\mathrm{X}$ & & \\
\hline Rhynchospora riparia (Nees) Boeckeler & 47772 & Castro, A. S. F. & 2357 & & Her & & & & & $\mathrm{X}$ & & \\
\hline Rhynchospora sp. & 49238 & Castro, A. S. F. & 2501 & & Her & & & & $\mathrm{X}$ & & & \\
\hline Scleria secans (L.) Urb. & 47771 & Castro, A. S. F. & 2356 & & Trep & & & & $\mathrm{X}$ & & & \\
\hline \multicolumn{13}{|l|}{ DILLENIACEAE } \\
\hline Curatella americana $\mathrm{L}$. & 41534 & Moro, M. F. & 339 & Cajueiro-bravo & Arv & & & & $\mathrm{X}$ & & & \\
\hline
\end{tabular}


Tabela 1. Continuação

\begin{tabular}{|c|c|c|c|c|c|c|c|c|c|c|c|}
\hline Espécie & EAC & Coletor & $\mathrm{NC}$ & Nome popular & Hábito & $\mathrm{P} 1$ & $\mathrm{P} 2$ & $\mathrm{D}$ & $\mathrm{T}$ & A & $\mathrm{C} \mathrm{M}$ \\
\hline Davilla cearensis Huber & 44638 & Ferreira, R. G. & 44 & Cipó-de-fogo & Trep & & & $\mathrm{X}$ & $\mathrm{X}$ & & \\
\hline Tetracera breyniana Schltdl. & 50017 & Magalhães, H. & 63 & Cipó-de-fogo & Trep & & & $\mathrm{X}$ & $\mathrm{X}$ & & \\
\hline Tetracera willdenowiana Steud. & 42553 & Moro, M. F. & 369 & Cipó-de-fogo & Trep & & & $\mathrm{X}$ & $\mathrm{X}$ & & \\
\hline \multicolumn{12}{|l|}{ DIOSCOREACEAE } \\
\hline $\begin{array}{l}\text { Dioscorea piperifolia Humb. \& Bonpl. ex Willd. } \\
\text { DROSERACEAE }\end{array}$ & 49132 & Castro, A. S. F. & 2489 & & Trep & & & \multicolumn{2}{|c|}{ DROSERACEAE } & & \\
\hline Drosera sessilifolia A. St.-Hil. & 49799 & Castro, A. S. F. & 2545 & & Her & & & & & $\mathrm{X}$ & \\
\hline \multicolumn{12}{|l|}{ EBENACEAE } \\
\hline Diospyros inconstans Jacq. & 42554 & Moro, M. F. & 384 & Fruta-de-cabra & Arb & & & & $\mathrm{X}$ & & \\
\hline \multicolumn{12}{|l|}{ ERIOCAULACEAE } \\
\hline Paepalanthus bifidus (Schrad.) Kunth & 47804 & Castro, A. S. F. & 2362 & & Her & & & & $\mathrm{X}$ & $\mathrm{X}$ & \\
\hline Paepalanthus sp. & 47805 & Castro, A. S. F. & 2361 & & Her & & & & $\mathrm{X}$ & $\mathrm{X}$ & \\
\hline \multicolumn{12}{|l|}{ ERYTHROXYLACEAE } \\
\hline Erythroxylum barbatum O.E.Schulz & 48839 & Moro, M. F. & 715 & & Arb & & & & $\mathrm{X}$ & & \\
\hline Erythroxylum laetevirens O.E.Schulz & 42546 & Moro, M. F. & 393 & & Arb & & & & $\mathrm{X}$ & & \\
\hline \multicolumn{12}{|l|}{ EUPHORBIACEAE } \\
\hline Astraea lobata (L.) Klotzsch & 34236 & Vieira, A. V. & sn & & Her & & & & $\mathrm{X}$ & & \\
\hline Cnidoscolus urens (L.) Arthur & 47402 & Castro, A. S. F. & 2343 & Cansanção & Arb & & $\mathrm{X}$ & $\mathrm{X}$ & $\mathrm{X}$ & & \\
\hline Croton anisodontus Müll. Arg. & 49024 & Castro, A. S. F. & 2371 & Marmeleiro-branco & Arb & & & & $\mathrm{X}$ & & \\
\hline Croton blanchetianus Baill. & 42580 & Moro, M. F. & 366 & Marmeleiro & Arb & & & & $\mathrm{X}$ & & $\mathrm{X}$ \\
\hline Croton echioides Baill. & 49029 & Castro, A. S. F. & 2454 & & Arb & & & & $\mathrm{X}$ & & \\
\hline Croton glandulosus L. & 34235 & Vieira, A. V. & sn & & Arb & & & & $\mathrm{X}$ & & \\
\hline Croton heliotropiifolius Kunth * & 39579 & Castro, A. S. F. & 1713 & Velame & Arb & & & & & & $\mathrm{X}$ \\
\hline Croton nepetifolius Baill. & 49134 & Castro, A. S. F. & 2491 & & Arb & & & & $\mathrm{X}$ & & \\
\hline Dalechampia brasiliensis Lam. & 41470 & Moro, M. F. & 303 & & Trep & & & & $\mathrm{X}$ & & \\
\hline Euphorbia hyssopifolia L. & 33751 & Lima, M. F. & sn & Leiteira & Her & & $\mathrm{X}$ & & $\mathrm{X}$ & & \\
\hline Jatropha mollissima (Pohl) Baill. & 47403 & Castro, A. S. F. & 2344 & Pinhão-bravo & Arb & & & $\mathrm{X}$ & $\mathrm{X}$ & & $\mathrm{X}$ \\
\hline Manihot tripartita (Spreng.) Müll. Arg. & 49165 & Castro, A. S. F. & 2497 & & Arb & & & & $\mathrm{X}$ & & \\
\hline Manihot sp. & & Observada & & & Trep & & & & $\mathrm{X}$ & & \\
\hline Microstachys corniculata (Vahl) Griseb. & 48172 & Castro, A. S. F. & 2399 & & Her & & & & $\mathrm{X}$ & $\mathrm{X}$ & \\
\hline Sapium argutum (Müll.Arg.) Huber * & 31377 & Castro, A. S. F. & 1171 & Burra-leiteira & Arv & & & & & & $\mathrm{X}$ \\
\hline \multicolumn{12}{|l|}{ FABACEAE-CAES } \\
\hline Apuleia leiocarpa (Vogel) J.F.Macbr. & 48176 & Castro, A. S. F. & 2403 & Jataí & Arv & & & $\mathrm{X}$ & $\mathrm{X}$ & & \\
\hline Bauhinia subclavata Benth. & 48160 & Castro, A. S. F. & 2387 & Mororó & Arv & & & & $\mathrm{X}$ & & \\
\hline Bauhinia ungulata L. & 41546 & Moro, M. F. & 265 & Mororó & Arv & & & & $\mathrm{X}$ & & $\mathrm{X}$ \\
\hline Chamaecrista calycioides (DC. ex Collad.) Greene & 34230 & Vieira, A. V. & sn & & Her & & $\mathrm{X}$ & & $\mathrm{X}$ & & \\
\hline Chamaecrista diphylla (L.) Greene & 42565 & Moro, M. F. & 350 & Mondubim & Her & & $\mathrm{X}$ & & $\mathrm{X}$ & & \\
\hline $\begin{array}{l}\text { Chamaecrista ensiformis (Vell.) H.S. Irwin \& } \\
\text { Barneby }\end{array}$ & 33074 & Lima, M. F. & sn & Pau-ferro & Arv & & & $\mathrm{X}$ & $\mathrm{X}$ & & \\
\hline Chamaecrista flexuosa (L.) Greene & 47398 & Castro, A. S. F. & 2339 & & Arb & & $\mathrm{X}$ & & $\mathrm{X}$ & & \\
\hline $\begin{array}{l}\text { Chamaecrista hispidula (Vahl) H.S. Irwin \& } \\
\text { Barneby }\end{array}$ & 47408 & Castro, A. S. F. & 2349 & & Her & & $\mathrm{X}$ & & $\mathrm{X}$ & & \\
\hline Copaifera arenicola (Ducke) J.Costa \& L.P. Queiroz & 31365 & Castro, A. S. F. & 1159 & Pau-d’óleo & Arv & & & $\mathrm{X}$ & $\mathrm{X}$ & & \\
\hline $\begin{array}{l}\text { Hymenaea courbaril var. stilbocarpa (Hayne) Y.T. } \\
\text { Lee \& Langenh. }\end{array}$ & 41502 & Moro, M. F. & 238 & Jatobá & Arv & & & $\mathrm{X}$ & $\mathrm{X}$ & & \\
\hline Poincianella bracteosa (Tul.) L.P. Queiroz & 44610 & Ferreira, R. G. & 10 & Catingueira & Arv & & & $\mathrm{X}$ & $\mathrm{X}$ & & $\mathrm{X}$ \\
\hline Senna alata (L.) Roxb. & 47802 & Castro, A. S. F. & 2364 & Manjerioba-do-Pará & Arb & & & & & $\mathrm{X}$ & \\
\hline $\begin{array}{l}\text { Senna macranthera (DC. ex Collad.) H.S. Irwin \& } \\
\text { Barneby }\end{array}$ & 41569 & Menezes, M. O. T. & 7 & & Arb & & & $\mathrm{X}$ & $\mathrm{X}$ & & \\
\hline Senna obtusifolia (L.) H.S. Irwin \& Barneby & 37780 & Roberto, I. J. & 6 & Mata-pasto & Arb & & & & $\mathrm{X}$ & & $\mathrm{X}$ \\
\hline Senna rizzinii H.S. Irwin \& Barneby & 33071 & Lima, M. F. & sn & & Arb & & & $\mathrm{X}$ & $\mathrm{X}$ & & \\
\hline Senna splendida (Vogel) H.S. Irwin \& Barneby & 41544 & Moro, M. F. & 268 & & Arb & & & $\mathrm{X}$ & $\mathrm{X}$ & & \\
\hline Senna trachypus (Benth.) H.S. Irwin \& Barneby & 49084 & Castro, A. S. F. & 2470 & & Arb & & & & $\mathrm{X}$ & & \\
\hline \multicolumn{12}{|l|}{ FABACEAE-FAB } \\
\hline Abrus precatorius $\mathrm{L}$. & 41486 & Moro, M. F. & 287 & Jiriquiti & Trep & & & $\mathrm{X}$ & $\mathrm{X}$ & & $\mathrm{X}$ \\
\hline Aeschynomene histrix Poir. * & 39661 & Castro, A. S. F. & 1748 & & Her & & $\mathrm{X}$ & & $\mathrm{X}$ & $\mathrm{X}$ & \\
\hline Aeschynomene marginata Benth. & 34224 & Vieira, A. V. & sn & & Her & & $\mathrm{X}$ & & $\mathrm{X}$ & $\mathrm{X}$ & \\
\hline Aeschynomene sensitiva Sw. & 41477 & Moro, M. F. & 310 & & Her & & $\mathrm{X}$ & & $\mathrm{X}$ & $\mathrm{X}$ & \\
\hline
\end{tabular}


Tabela 1. Continuação

\begin{tabular}{|c|c|c|c|c|c|c|c|c|c|c|c|}
\hline Espécie & EAC & Coletor & NC & Nome popular & Hábito & P1 & $\mathrm{P} 2$ & $\mathrm{D}$ & $\mathrm{T}$ & A & C $\mathrm{N}$ \\
\hline Aeschynomene viscidula Michx. & 47407 & Castro, A. S. F. & 2348 & & Her & & $\mathrm{X}$ & & $\mathrm{X}$ & $\mathrm{X}$ & \\
\hline Andira surinamensis (Bondt) Splitg. ex Amshoff & 41468 & Moro, M. F. & 293 & Angelim & Arv & & & & $\mathrm{x}$ & & $\mathrm{X}$ \\
\hline Canavalia rosea (Sw.) DC. & 33749 & Vieira, A. V. & sn & Fava-de-boi & Trep & $\mathrm{X}$ & $\mathrm{X}$ & & & & \\
\hline Centrosema brasilianum (L.) Benth. & 41572 & Menezes, M. O. T. & 9 & & Trep & & $\mathrm{X}$ & & $\mathrm{X}$ & $\mathrm{X}$ & \\
\hline Centrosema rotundifolium Mart. ex Benth. & 34225 & Vieira, A. V. & sn & & Trep & & $\mathrm{X}$ & & & & \\
\hline Clitoria laurifolia Poir. & 41478 & Moro, M. F. & 311 & & Arb & & $\mathrm{X}$ & & $\mathrm{X}$ & $\mathrm{X}$ & \\
\hline Crotalaria retusa L. (invasora) & 33531 & Vieira, A. V. & sn & Chocalho-de-cobra & Arb & & $\mathrm{X}$ & & $\mathrm{X}$ & $\mathrm{X}$ & \\
\hline Dalbergia ecastaphyllum (L.) Taub. & 42571 & Moro, M. F. & 347 & Bugi & Arb & & & & $\mathrm{X}$ & & X \\
\hline Desmodium barbatum (L.) Benth. * & 39656 & Castro, A. S. F. & 1743 & & Her & & $\mathrm{X}$ & & $\mathrm{X}$ & $\mathrm{X}$ & \\
\hline Desmodium glabrum (Mill.) DC. & 48154 & Castro, A. S. F. & 2381 & & Her & & & & $\mathrm{X}$ & & \\
\hline Dioclea lasiophylla Mart. ex Benth. & 41516 & Moro, M. F. & 342 & & Trep & & & $\mathrm{X}$ & $\mathrm{X}$ & $\mathrm{X}$ & \\
\hline Dioclea violacea Mart. ex Benth. & 49167 & Castro, A. S. F. & 2499 & Mucunã & Trep & & & $\mathrm{X}$ & $\mathrm{X}$ & & \\
\hline Galactia striata (Jacq.) Urb. & 48025 & Castro, A. S. F. & 2376 & & Trep & & & $\mathrm{X}$ & $\mathrm{X}$ & & \\
\hline Indigofera hirsuta L. (invasora) & 33752 & Vieira, A. V. & sn & & Arb & & $\mathrm{X}$ & & $\mathrm{X}$ & & \\
\hline Indigofera microcarpa Desv. & 33561 & Vieira, A. V. & sn & & Arb & & $\mathrm{x}$ & & $\mathrm{x}$ & & \\
\hline Indigofera suffruticosa Mill. & 47404 & Castro, A. S. F. & 2345 & & Arb & & & & $\mathrm{x}$ & $\mathrm{X}$ & \\
\hline Leptolobium dasycarpum Vogel & 48834 & Moro, M. F. & 722 & Milhome & Arv & & & & $\mathrm{x}$ & & \\
\hline Lonchocarpus araripensis Benth. & 48321 & Ferreira, R. G. & sn & & Arv & & & & $\mathrm{x}$ & & \\
\hline Luetzelburgia auriculata (Allemão) Ducke & 48159 & Castro, A. S. F. & 2386 & Pau-mocó & Arv & & & & $\mathrm{X}$ & & \\
\hline $\begin{array}{l}\text { Macroptilium atropurpureum (Sessé \& Moc. ex } \\
\text { DC.) Urb. (invasora) }\end{array}$ & 33746 & Vieira, A. V. & sn & & Trep & & & & $\mathrm{X}$ & & \\
\hline Ormosia fastigiata Tul. & 48185 & Castro, A. S. F. & 2412 & Cajarana-brava & Arv & & & & $\mathrm{X}$ & & \\
\hline Rhynchosia phaseoloides (Sw.) DC. & 50283 & Castro, A. S. F. & 2551 & & Trep & & & & $\mathrm{x}$ & & \\
\hline Stylosanthes angustifolia Vogel * & 39710 & Castro, A. S. F. & 1800 & & Her & & $\mathrm{X}$ & & $\mathrm{x}$ & $\mathrm{X}$ & \\
\hline Stylosanthes capitata Vogel & 41557 & Menezes, M. O. T. & 23 & & Her & & $\mathrm{X}$ & & $\mathrm{X}$ & $\mathrm{x}$ & \\
\hline Stylosanthes humilis Kunth & 50162 & Magalhães, H. & 3 & & Her & & & $\mathrm{X}$ & & $\mathrm{x}$ & \\
\hline Stylosanthes viscosa (L.) Sw. * & 39667 & Castro, A. S. F. & 1754 & & Her & & $\mathrm{X}$ & & $\mathrm{X}$ & $\mathrm{X}$ & \\
\hline Tephrosia egregia Sandwith * & 49041 & Castro, A. S. F. & 2466 & & Subarb & & $\mathrm{X}$ & & $\mathrm{x}$ & & \\
\hline Tephrosia purpurea (L.) Pers. & 33750 & Vieira, A. V. & sn & Anil & Her & & & & $\mathrm{x}$ & & \\
\hline Vatairea macrocarpa (Benth.) Ducke & 48163 & Castro, A. S. F. & 2390 & Angelim & Arv & & & & $\mathrm{x}$ & & \\
\hline Vigna luteola (Jacq.) Benth. & 41532 & Moro, M. F. & 314 & & Trep & & $\mathrm{X}$ & & $\mathrm{X}$ & $\mathrm{x}$ & \\
\hline Vigna peduncularis (Kunth) Fawc.\& Rendle & 48167 & Castro, A. S. F. & 2394 & & Trep & & $\mathrm{X}$ & & $\mathrm{X}$ & $\mathrm{x}$ & \\
\hline Zornia latifolia Sm. & 34229 & Vieira, A. V. & sn & & Her & & $\mathrm{X}$ & & $\mathrm{X}$ & $\mathrm{x}$ & \\
\hline \multicolumn{12}{|l|}{ FABACEAE-MIM } \\
\hline Calliandra sessilis Benth. & 41539 & Moro, M. F. & 275 & Barba-de-soim & Arb & & & & $\mathrm{x}$ & & \\
\hline $\begin{array}{l}\text { Chloroleucon acacioides (Ducke) Barneby \& } \\
\text { J.W.Grimes }\end{array}$ & 33072 & Lima, M. F. & sn & Jurema-branca & Arv & & & $\mathrm{X}$ & $\mathrm{X}$ & & \\
\hline Enterolobium timbouva Mart. ${ }^{*}$ & 39730 & Castro, A. S. F. & 1815 & Timbaúba & Arv & & & $\mathrm{X}$ & $\mathrm{X}$ & & \\
\hline Mimosa arenosa (Willd.) Poir. & 49126 & Castro, A. S. F. & 2483 & Tiririca & Arv & & & $\mathrm{d}$ & $\mathrm{d}$ & & \\
\hline Mimosa caesalpiniifolia Benth. & 48182 & Castro, A. S. F. & 2409 & Sabiá & Arv & & & $\mathrm{d}$ & $\mathrm{d}$ & & $\mathrm{X}$ \\
\hline Mimosa hirsutissima Mart. & 34220 & Vieira, A. V. & sn & & Her & & & & $\mathrm{X}$ & $\mathrm{X}$ & \\
\hline Mimosa misera Benth. & 41566 & Menezes, M. O. T. & 4 & & Subarb & & & $\mathrm{X}$ & $\mathrm{X}$ & $\mathrm{X}$ & \\
\hline Mimosa quadrivalvis var. leptocarpa (DC.) Barneby & 50150 & Magalhães, H. & 5 & Malícia & Subarb & & & & $\mathrm{X}$ & $\mathrm{x}$ & \\
\hline Mimosa sensitiva $\mathrm{L}$ & 41556 & Menezes, M. O. T. & 10 & Malícia-de-boi & Arb & & & $\mathrm{X}$ & $\mathrm{X}$ & $\mathrm{x}$ & \\
\hline Mimosa tenuiflora (Willd.) Poir. & 47395 & Castro, A. S. F. & 2337 & Jurema-preta & Arv & & & & $\mathrm{d}$ & & $\mathrm{X}$ \\
\hline Neptunia oleracea Lour. & 41485 & Moro, M. F. & 282 & & Her & & & & & $\mathrm{X}$ & \\
\hline Neptunia plena (L.) Benth. & 49240 & Castro, A. S. F. & 2503 & & Subarb & & & & & $\mathrm{X}$ & \\
\hline Parkia platycephala Benth. & 46034 & Ferreira, R. G. & 116 & Visgueiro & Arv & & & & $\mathrm{X}$ & & \\
\hline Piptadenia stipulacea (Benth.) Ducke & 41471 & Moro, M. F. & 304 & Jurema-branca & Arv & & & $\mathrm{d}$ & $\mathrm{d}$ & & $\mathrm{x}$ \\
\hline $\begin{array}{l}\text { Pityrocarpa moniliformis (Benth.) Luckow \& } \\
\text { R.W.Jobson }\end{array}$ & 42583 & Moro, M. F. & 362 & Catanduba & Arv & & & & $\mathrm{X}$ & & \\
\hline Plathymenia reticulata Benth. & 42561 & Moro, M. F. & 390 & Acende-candeia & Arv & & & & $\mathrm{x}$ & & \\
\hline \multicolumn{12}{|l|}{ GENTIANACEAE } \\
\hline Curtia tenuifolia (Aubl.) Knobl. & 49800 & Castro, A. S. F. & 2546 & & Her & & & & & $\mathrm{X}$ & \\
\hline Voyria aphylla (Jacq.) Pers. & 42540 & Moro, M. F. & 375 & & Her & & & & $\mathrm{X}$ & & \\
\hline \multicolumn{12}{|l|}{ HELICONIACEAE } \\
\hline Heliconia psittacorum L.f. & 49088 & Castro, A. S. F. & 2474 & Pacavira & Her & & & & & $\mathrm{X}$ & \\
\hline HUMIRIACEAE & & & & & & & & & & & \\
\hline
\end{tabular}


Tabela 1. Continuação

\begin{tabular}{|c|c|c|c|c|c|c|c|c|c|c|c|}
\hline Espécie & EAC & Coletor & NC & Nome popular & Hábito & $\mathrm{P} 1$ & P2 & $\mathrm{D}$ & $\mathrm{T}$ & A & C $\mathrm{M}$ \\
\hline Humiria balsamifera (Aubl.) J.St.-Hil. & 42551 & Moro, M. F. & 378 & Mangue & Arv & & & & $\mathrm{X}$ & & \\
\hline \multicolumn{12}{|l|}{ HYDROLEACEAE } \\
\hline Hydrolea spinosa $\mathrm{L}$. & 48181 & Castro, A. S. F. & 2408 & & Subarb & & & & & $\mathrm{X}$ & \\
\hline \multicolumn{12}{|l|}{ HYPERICACEAE } \\
\hline Vismia guianensis (Aubl.) Choisy & 49658 & Ferreira, R. G. & sn & Lacre & Arb & & & & $\mathrm{X}$ & & \\
\hline \multicolumn{12}{|l|}{ KRAMERIACEAE } \\
\hline Krameria tomentosa A.St.-Hil. & 41582 & Menezes, M. O. T. & 17 & Carrapicho & Subarb & & & & $\mathrm{X}$ & & \\
\hline \multicolumn{12}{|l|}{ LAMIACEAE } \\
\hline Amasonia campestris (Aubl.) Moldenke & 42555 & Moro, M. F. & 368 & Flor-de-urubu & Subarb & & & & $\mathrm{X}$ & & \\
\hline Eriopidion strictum (Benth.) Harley * & 39762 & Castro, A. S. F. & 1844 & & Her & & & & $\mathrm{X}$ & & \\
\hline Hyptis suaveolens Poit. & 41562 & Menezes, M. O. T. & 13 & Bamburral & Subarb & & & & $\mathrm{X}$ & & $\mathrm{X}$ \\
\hline Marsypianthes chamaedrys (Vahl) Kuntze & 42548 & Moro, M. F. & 387 & & Her & & $\mathrm{X}$ & & $\mathrm{X}$ & $\mathrm{X}$ & \\
\hline \multicolumn{12}{|l|}{ LAURACEAE } \\
\hline Cassytha filiformis $\mathrm{L}$. & 41558 & Menezes, M. O. T. & 20 & Erva-de-chumbo & Paras & & $\mathrm{X}$ & $\mathrm{X}$ & $\mathrm{X}$ & & \\
\hline Ocotea canaliculata (Rich.) Mez & 41490 & Moro, M. F. & 254 & Louro & Arv & & & & $\mathrm{X}$ & & \\
\hline \multicolumn{12}{|l|}{ LENTIBULARIACEAE } \\
\hline Utricularia simulans Pilg. & 49239 & Castro, A. S. F. & 2502 & & Her & & & & & $\mathrm{X}$ & \\
\hline \multicolumn{12}{|l|}{ LINDERNIACEAE } \\
\hline Lindernia rotundifolia (L.) Alston & 41522 & Moro, M. F. & 333 & & Her & & & & & $\mathrm{X}$ & \\
\hline \multicolumn{12}{|l|}{ LOGANIACEAE } \\
\hline Strychnos parvifolia A.DC. & 46027 & Ferreira, R. G. & 112 & Gulari & Arb & & & $\mathrm{X}$ & $\mathrm{X}$ & & \\
\hline \multicolumn{12}{|l|}{ LYTHRACEAE } \\
\hline Cuphea campestris Koehne & 50165 & Magalhães, H. & 232 & & Her & & & $\mathrm{X}$ & $\mathrm{X}$ & $\mathrm{X}$ & \\
\hline Lafoensia vandelliana Cham. \& Schltdl. & 47775 & Castro, A. S. F. & 2369 & & Arv & & & & $\mathrm{X}$ & & \\
\hline \multicolumn{12}{|l|}{ MALPIGHIACEAE } \\
\hline Bunchosia apiculata Huber & 49995 & Magalhães, H. & 163 & & Arb & & & $\mathrm{X}$ & $\mathrm{X}$ & & \\
\hline Byrsonima crassifolia (L.) Kunth & 41527 & Moro, M. F. & 320 & Murici & Arb-Arv & & $\mathrm{X}$ & $\mathrm{X}$ & $\mathrm{X}$ & & \\
\hline Byrsonima gardneriana A.Juss. & 42552 & Moro, M. F. & 382 & Murici-pitanga & Arv & & & $\mathrm{X}$ & $\mathrm{X}$ & & \\
\hline Byrsonima spicata (Cav.) DC. & 48184 & Castro, A. S. F. & 2411 & Murici & Arb & & & $\mathrm{X}$ & $\mathrm{X}$ & & \\
\hline $\begin{array}{l}\text { Diplopterys lutea (Griseb.) W.R.Anderson \& } \\
\text { C.C.Davis }\end{array}$ & 33559 & Vieira, A. V. & sn & & Trep & & & $\mathrm{X}$ & $\mathrm{X}$ & & \\
\hline $\begin{array}{l}\text { Diplopterys pubipetala (A.Juss.) W.R.Anderson \& } \\
\text { C.C.Davis }\end{array}$ & 33081 & Lima, M. F. & sn & & Trep & & & $\mathrm{X}$ & $\mathrm{X}$ & & \\
\hline Stigmaphyllon paralias A.Juss. & 42578 & Moro, M. F. & 381 & & Subarb & & & $\mathrm{X}$ & $\mathrm{X}$ & & \\
\hline \multicolumn{12}{|l|}{ MALVACEAE } \\
\hline Ayenia erecta Mart. \& K. Schum. & 49163 & Castro, A. S. F. & 2495 & & Her & & & & $\mathrm{X}$ & & \\
\hline Briquetia spicata (Kunth) Fryxell & 49128 & Castro, A. S. F. & 2485 & & Subarb & & & & $\mathrm{d}$ & & \\
\hline Helicteres heptandra L.B.Sm. & 41517 & Moro, M. F. & 340 & Guaxuma & Arb & & & & $\mathrm{X}$ & & $\mathrm{X}$ \\
\hline Luehea sp. & 41540 & Moro, M. F. & 274 & Açoita-cavalo & Arv & & & & $\mathrm{X}$ & & $\mathrm{X}$ \\
\hline Melochia betonicifolia A.St.-Hil. & 42557 & Moro, M. F. & 330 & & Subarb & & & $\mathrm{X}$ & $\mathrm{X}$ & & \\
\hline Pachira stenopetala Casar. & 48166 & Castro, A. S. F. & 2393 & Barriguda & Arv & & & $\mathrm{X}$ & $\mathrm{X}$ & & \\
\hline Pavonia cancellata (L.) Cav. & 34238 & Vieira, A. V. & sn & & Her & & $\mathrm{X}$ & & $\mathrm{X}$ & $\mathrm{X}$ & \\
\hline Sida anomala A.St.-Hil. & 34219 & Vieira, A. V. & sn & & Her & & $\mathrm{X}$ & & $\mathrm{X}$ & & \\
\hline Sida linifolia Cav. & 50176 & Magalhães, H. & 227 & & Her & & & $\mathrm{X}$ & $\mathrm{X}$ & & \\
\hline Sida salviifolia C. Presl & 48155 & Castro, A. S. F. & 2382 & & Her & & & & $\mathrm{X}$ & & \\
\hline Sterculia striata A.St.-Hil. \& Naudin & 44649 & Ferreira, R. G. & 60 & Xixá & Arv & & & $\mathrm{X}$ & $\mathrm{X}$ & & \\
\hline Triumfetta rhomboidea Jacq. * & 49042 & Castro, A. S. F. & 2467 & & Subarb & & & & $\mathrm{X}$ & $\mathrm{X}$ & \\
\hline Urena lobata $\mathrm{L}$. & 47391 & Castro, A. S. F. & 2332 & & Subarb & & & & $\mathrm{X}$ & $\mathrm{X}$ & \\
\hline Waltheria americana $\mathrm{L}$. & 41565 & Menezes, M. O. T. & 2 & Malva & Subarb & & & & $\mathrm{X}$ & $\mathrm{X}$ & \\
\hline \multicolumn{12}{|l|}{ MARANTACEAE } \\
\hline Maranta cf. sobolifera L. Andersson & 49026 & Castro, A. S. F. & 2451 & Araruta & Her & & & & & $\mathrm{X}$ & \\
\hline \multicolumn{12}{|l|}{ MELASTOMATACEAE } \\
\hline Comolia villosa (Aubl.) Triana & 39641 & Castro, A. S. F. & 1728 & & Her & & & & & $\mathrm{X}$ & \\
\hline Mouriri cearensis Huber & 43974 & Sousa, T. M. & sn & Puçá & Arv & & & $\mathrm{X}$ & $\mathrm{X}$ & & \\
\hline Mouriri guianensis Aubl. & 41483 & Moro, M. F. & 277 & Gurguri & Arv & & & & & & $\mathrm{X}$ \\
\hline Pterolepis glomerata (Rottb.) Miq. & 41520 & Moro, M. F. & 335 & & Her & & & & & $\mathrm{X}$ & \\
\hline MORACEAE & & & & & & & & & & & \\
\hline
\end{tabular}


Tabela 1. Continuação

\begin{tabular}{|c|c|c|c|c|c|c|c|c|c|c|c|}
\hline Espécie & EAC & Coletor & NC & Nome popular & Hábito & P1 & P2 & $\mathrm{D}$ & $\mathrm{T}$ & $\mathrm{AC}$ & C $\mathrm{M}$ \\
\hline Brosimum gaudichaudii Trécul & 48318 & Ferreira, R. G. & sn & Inharé & Arv & & & & $\mathrm{X}$ & & \\
\hline Ficus elliotiana S.Moore * & 48029 & Castro, A. S. F. & 2380 & Gameleira & Arv & & & & $\mathrm{X}$ & $\mathrm{X}$ & $\mathrm{X}$ \\
\hline Ficus enormis Mart. ex Miq. & 31378 & Castro, A. S. F. & 1172 & Gameleira & Arv & & & $\mathrm{x}$ & $\mathrm{X}$ & & \\
\hline Ficus sp. & 41535 & Moro, M. F. & 341 & Gameleira & Arv & & & $\mathrm{x}$ & $\mathrm{X}$ & & \\
\hline Maclura tinctoria (L.) D.Don ex Steud. & 41624 & Moro, M. F. & 272 & Tatajuba & Arv & & & $\mathrm{X}$ & $\mathrm{X}$ & & \\
\hline \multicolumn{12}{|l|}{ MYRTACEAE } \\
\hline Campomanesia aromatica (Aubl.) Griseb. & 48833 & Moro, M. F. & 721 & Guabiraba & Arv & & & $\mathrm{x}$ & $\mathrm{X}$ & & \\
\hline $\begin{array}{l}\text { Eugenia luschnathiana (O.Berg) Klotzsch ex } \\
\text { B.D.Jacks. }\end{array}$ & 41472 & Moro, M. F. & 305 & Ubaia & Arv & & & $\mathrm{X}$ & $\mathrm{X}$ & & \\
\hline Eugenia punicifolia (Kunth) DC. & 41570 & Menezes, M. O. T. & 1 & Murta & Arb & & & $\mathrm{X}$ & $\mathrm{X}$ & & \\
\hline Eugenia stictopetala DC. & 33073 & Lima, M. F. & sn & & Arv & & & $\mathrm{X}$ & $\mathrm{X}$ & & \\
\hline Eugenia sp.1 & 41473 & Moro, M. F. & 306 & Ubaia-de-raposa & Arb & & & & $\mathrm{X}$ & & \\
\hline Eugenia sp. 2 & 48831 & Moro, M. F. & 727 & Ubaitinga & Arv & & & $\mathrm{X}$ & $\mathrm{X}$ & & \\
\hline Myrcia guianensis (Aubl.) DC. & 41577 & Menezes, M. O. T. & 26 & Batinga & Arv & & & & $\mathrm{X}$ & & \\
\hline Myrcia multiflora (Lam.) DC. & 41484 & Moro, M. F. & 280 & Arrebenta-boi & Arv & & & & $\mathrm{X}$ & & \\
\hline Myrcia splendens (Sw.) DC. & 44675 & Ferreira, R. G. & 88 & Viuvinha & Arv & & & $\mathrm{X}$ & $\mathrm{X}$ & & \\
\hline Myrcia sp. & 49732 & Castro, A. S. F. & 2542 & Mapirunga & Arv & & & & $\mathrm{X}$ & & \\
\hline Myrciaria cuspidata O.Berg & 42562 & Moro, M. F. & 383 & & Arv & & & $\mathrm{x}$ & $\mathrm{X}$ & & \\
\hline Psidium guineense $\mathrm{Sw}$. & 41571 & Menezes, M. O. T. & 8 & Araçá & Arv & & & $\mathrm{x}$ & $\mathrm{X}$ & & \\
\hline Psidium sartorianum (O.Berg) Nied. & 48022 & Castro, A. S. F. & 2373 & Goiabinha & Arv & & & & $\mathrm{X}$ & & \\
\hline Syzygium cumini (L.) Skeels (invasora) & 48850 & Ferreira, R. G. & sn & Azeitona-roxa & Arv & & & & $\mathrm{X}$ & $\mathrm{X}$ & \\
\hline Myrtaceae Indeterminada & & Observada & & & Arv & & & & $\mathrm{X}$ & & \\
\hline \multicolumn{12}{|l|}{ NYCTAGINACEAE } \\
\hline Guapira laxa (Netto) Furlan & 49091 & Castro, A. S. F. & 2477 & João-mole & Arv & & & $\mathrm{X}$ & $\mathrm{X}$ & & \\
\hline Guapira nitida (Mart. ex J.A.Schmidt) Lundell & 44944 & Moro, M. F. & 707 & João-mole-preto & Arv & & & & $\mathrm{X}$ & & \\
\hline \multicolumn{12}{|l|}{ NYMPHAEACEAE } \\
\hline Nymphaea amazonum Mart. \& Zucc. & 47411 & Castro, A. S. F. & 2352 & & Her & & & & & $\mathrm{X}$ & \\
\hline Nymphaea tenerinervia Casp. & 49162 & Castro, A. S. F. & 2494 & & Her & & & & & $\mathrm{x}$ & \\
\hline \multicolumn{12}{|l|}{ OCHNACEAE } \\
\hline Ouratea sp. & 48822 & Moro, M. F. & 718 & Batiputá & Arv-Arb & & & $\mathrm{X}$ & $\mathrm{X}$ & & \\
\hline \multicolumn{12}{|l|}{ OLACACEAE } \\
\hline Ximenia americana $\mathrm{L}$. & 41564 & Menezes, M. O. T. & 12 & Ameixa & Arb-Arv & & & $\mathrm{X}$ & $\mathrm{X}$ & & \\
\hline \multicolumn{12}{|l|}{ ONAGRACEAE } \\
\hline Ludwigia leptocarpa (Nutt.) H.Hara & 41475 & Moro, M. F. & 308 & & Subarb & & & & & $\mathrm{X}$ & \\
\hline \multicolumn{12}{|l|}{ OPILIACEAE } \\
\hline Agonandra brasiliensis Miers ex Benth. \& Hook.f. & 46031 & Ferreira, R. G. & 117 & Marfim & Arv & & & & $\mathrm{x}$ & & \\
\hline \multicolumn{12}{|l|}{ ORCHIDACEAE } \\
\hline Epidendrum ciliare $\mathrm{L}$. & 31354 & Castro, A. S. F. & 1175 & & Epif & & & $\mathrm{X}$ & $\mathrm{X}$ & & \\
\hline Cyrtopodium holstii L.C.Menezes & 48836 & Moro, M. F. & 724 & Rabo-de-tatu & Her & & & $\mathrm{X}$ & $\mathrm{x}$ & & \\
\hline Oeceoclades maculata (Lindl.) Lindl. (invasora) & 42559 & Moro, M. F. & 323 & & Her & & & $\mathrm{X}$ & $\mathrm{X}$ & & \\
\hline \multicolumn{12}{|l|}{ PASSIFLORACEAE } \\
\hline Passiflora foetida $\mathrm{L}$. & 33535 & Vieira, A. V. & sn & & Trep & & $\mathrm{X}$ & $\mathrm{x}$ & $\mathrm{X}$ & $\mathrm{X}$ & \\
\hline Passiflora picturata Ker Gawl. & 47393 & Castro, A. S. F. & 2334 & Maracujá-do-mato & Trep & & & $\mathrm{x}$ & $\mathrm{X}$ & & \\
\hline Passiflora subrotunda Mast. & 41550 & Moro, M. F. & 256 & Maracujá-do-mato & Trep & & & $\mathrm{X}$ & $\mathrm{X}$ & & \\
\hline \multicolumn{12}{|l|}{ PHYLLANTHACEAE } \\
\hline Phyllanthus niruri L. & 50161 & Magalhães, H. & 235 & & Her & & & $\mathrm{X}$ & & & \\
\hline \multicolumn{12}{|l|}{ PLANTAGINACEAE } \\
\hline Bacopa cochlearia (Huber) L.B. Sm. & 49731 & Castro, A. S. F. & 2541 & & Her & & & & & $\mathrm{X}$ & \\
\hline Tetraulacium veroniciforme Turcz. & 49164 & Castro, A. S. F. & 2496 & & Her & & & & $\mathrm{X}$ & $\mathrm{X}$ & \\
\hline \multicolumn{12}{|l|}{ POACEAE } \\
\hline Andropogon fastigiatus Sw. & 47400 & Castro, A. S. F. & 2341 & & Her & & & $\mathrm{X}$ & $\mathrm{X}$ & $\mathrm{x}$ & \\
\hline Aristida longifolia Trin. & 47773 & Castro, A. S. F. & 2358 & & Her & & & & $\mathrm{X}$ & & \\
\hline Axonopus sp. & 49089 & Castro, A. S. F. & 2475 & & Her & & & & $\mathrm{X}$ & & \\
\hline $\begin{array}{l}\text { Digitaria bicornis (Lam.) Roem. \& Schult. } \\
\text { (invasora) }\end{array}$ & 49978 & Magalhães, H. & 265 & & Her & & & $\mathrm{x}$ & $\mathrm{x}$ & & \\
\hline Eragrostis ciliaris (L.) R.Br. & 48027 & Castro, A. S. F. & 2378 & & Her & & $\mathrm{X}$ & & $\mathrm{X}$ & & \\
\hline Eragrostis maypurensis (Kunth) Steud. & 48026 & Castro, A. S. F. & 2377 & & Her & & $\mathrm{X}$ & & $\mathrm{X}$ & & \\
\hline
\end{tabular}


Tabela 1. Continuação

\begin{tabular}{|c|c|c|c|c|c|c|c|c|c|c|c|c|}
\hline Espécie & EAC & Coletor & NC & Nome popular & Hábito & P1 & $\mathrm{P} 2$ & $\mathrm{D}$ & $\mathrm{T}$ & A & $\mathrm{C} \quad 1$ & $\mathrm{M}$ \\
\hline Eragrostis rufescens Schrad. ex Schult. * & 39658 & Castro, A. S. F. & 1745 & & Her & & $\mathrm{X}$ & & $\mathrm{x}$ & & & \\
\hline Gymnopogon foliosus (Willd.) Nees & 48024 & Castro, A. S. F. & 2375 & & Her & & $\mathrm{X}$ & & $\mathrm{x}$ & & & \\
\hline Melinis repens (Willd.) Zizka (invasora) & 34240 & Vieira, A. V. & sn & & Her & & $\mathrm{X}$ & & $\mathrm{X}$ & & & \\
\hline Paspalum maritimum Trin. & 33744 & Vieira, A. V. & sn & Capim-gengibre & Her & & $\mathrm{X}$ & $\mathrm{X}$ & $\mathrm{x}$ & & & \\
\hline Paspalum scutatum Nees ex Trin. ${ }^{*}$ & 38214 & Castro, A. S. F. & 1602 & & Her & & & & $\mathrm{X}$ & & & \\
\hline Paspalum vaginatum $\mathrm{Sw}$. & 49131 & Castro, A. S. F. & 2488 & & Her & $\mathrm{X}$ & $\mathrm{X}$ & & & $\mathrm{X}$ & & \\
\hline Reimarochloa brasiliensis (Spreng.) Hitchc. * & 38213 & Castro, A. S. F. & 1601 & & Her & & $\mathrm{X}$ & & $\mathrm{x}$ & & & \\
\hline Schizachyrium condensatum (Kunth) Nees & 48174 & Castro, A. S. F. & 2401 & Rabo-de-raposa & Her & & & & $\mathrm{X}$ & $\mathrm{X}$ & & \\
\hline Sporobolus virginicus (L.) Kunth & 42570 & Moro, M. F. & 353 & & Her & $\mathrm{X}$ & $\mathrm{X}$ & & & & & \\
\hline Streptostachys asperifolia Desv. & 47390 & Castro, A. S. F. & 2331 & & Her & & & $\mathrm{X}$ & $\mathrm{x}$ & & & \\
\hline Trachypogon spicatus (L.f.) Kuntze & 49079 & Castro, A. S. F. & 2359 & & Her & & & & $\mathrm{x}$ & & & \\
\hline \multicolumn{13}{|l|}{ POLYGALACEAE } \\
\hline $\begin{array}{l}\text { Bredemeyera laurifolia (A.St.-Hil.) Klotzsch ex } \\
\text { A.W.Benn. }\end{array}$ & 41542 & Moro, M. F. & 269 & Pacari & Trep & & & & $\mathrm{X}$ & & & \\
\hline Polygala martiana A.W.Benn. & 34231 & Vieira, A. V. & sn & & Her & & $\mathrm{X}$ & & $\mathrm{X}$ & $\mathrm{X}$ & & \\
\hline Polygala trichosperma Jacq. & 42541 & Moro, M. F. & 386 & & Her & & & & $\mathrm{x}$ & $\mathrm{X}$ & & \\
\hline \multicolumn{13}{|l|}{ POLYGONACEAE } \\
\hline Coccoloba latifolia Lam. & 41481 & Moro, M. F. & 279 & Coaçu & Arb & & & & $\mathrm{x}$ & & $\mathrm{X}$ & \\
\hline Coccoloba obtusifolia Jacq. * & 31372 & Castro, A. S. F. & 1166 & & Arb & & & & & & $\mathrm{X}$ & \\
\hline Coccoloba ramosissima Wedd. & 42568 & Moro, M. F. & 372 & Carrasco & Arb & & & $\mathrm{X}$ & $\mathrm{x}$ & & & \\
\hline Coccoloba sp. & 50048 & Magalhães, H. & 132 & & Trep & & & $\mathrm{X}$ & $\mathrm{X}$ & & & \\
\hline \multicolumn{13}{|l|}{ PONTEDERIACEAE } \\
\hline Pontederia cordata L. & 42544 & Moro, M. F. & 283 & Pacavira & Her & & & & & $\mathrm{X}$ & & \\
\hline \multicolumn{13}{|l|}{ PORTULACACEAE } \\
\hline Portulaca pilosa $\mathrm{L}$. & 49033 & Castro, A. S. F. & 2458 & & Her & & $\mathrm{X}$ & & $\mathrm{X}$ & $\mathrm{X}$ & & \\
\hline \multicolumn{13}{|l|}{ RHAMNACEAE } \\
\hline Gouania virgata Reissek & 49130 & Castro, A. S. F. & 2487 & & Trep & & & & $\mathrm{x}$ & & & \\
\hline Ziziphus platyphylla Reissek & 41541 & Moro, M. F. & 271 & Juazeiro & Arv & & & $\mathrm{X}$ & $\mathrm{x}$ & & & \\
\hline \multicolumn{13}{|l|}{ RHIZOPHORACEAE } \\
\hline Rhizophora mangle L. & 42582 & Moro, M. F. & 351 & Mangue-vermelho & Arv & & & & & & & $\mathrm{x}$ \\
\hline \multicolumn{13}{|l|}{ RUBIACEAE } \\
\hline Alseis pickelii Pilg. \& Schmale & 48165 & Castro, A. S. F. & 2392 & Cocão & Arv & & & & $\mathrm{x}$ & & & \\
\hline Duroia sp. & 44943 & Moro, M. F. & 708 & Canelão & Arv & & & & $\mathrm{x}$ & & & \\
\hline Borreria verticillata (L.) G.Mey. & 41529 & Moro, M. F. & 317 & Vassourinha-de-botão & Arb & & $\mathrm{X}$ & & $\mathrm{X}$ & $\mathrm{X}$ & & \\
\hline Chiococca alba (L.) Hitchc. & 31375 & Castro, A. S. F. & 1169 & Caninana & Arb & & & $\mathrm{X}$ & $\mathrm{x}$ & & & \\
\hline Chomelia obtusa Cham. \& Schltdl. & 42579 & Moro, M. F. & 357 & Cruzeta & Arb & & & & & & $\mathrm{X}$ & \\
\hline Cordiera rigida (K.Schum.) Kuntze & 41525 & Moro, M. F. & 324 & Sogro & Arb & & & & $\mathrm{X}$ & & & \\
\hline Cordiera sessilis (Vell.) Kuntze & 41509 & Moro, M. F. & 244 & Canela-de-veado & Arb & & & $\mathrm{X}$ & $\mathrm{x}$ & & & \\
\hline $\begin{array}{l}\text { Diodella gardneri (K.Schum.) Bacigalupo \& } \\
\text { E.L.Cabral }\end{array}$ & 33562 & Vieira, A. V. & sn & & Arb & & & $\mathrm{X}$ & $\mathrm{x}$ & $\mathrm{X}$ & & \\
\hline Diodella teres (Walter) Small & 34233 & Vieira, A. V. & sn & & Arb & & & & $\mathrm{x}$ & $\mathrm{X}$ & & \\
\hline Faramea nitida Benth. & 42542 & Moro, M. F. & 376 & Farinha-seca & Arb & & & & $\mathrm{x}$ & & & \\
\hline Genipa americana $\mathrm{L}$. & 48560 & Castro, A. S. F. & 2417 & Jenipapo & Arv & & & & $\mathrm{x}$ & & $\mathrm{X}$ & \\
\hline Guettarda aff. angelica Mart. ex Müll.Arg. & 31374 & Castro, A. S. F. & 1168 & & Arb & & & & $\mathrm{x}$ & & $\mathrm{X}$ & \\
\hline Guettarda angelica Mart. ex Müll.Arg. * & 39729 & Castro, A. S. F. & 1814 & Angélica & Arb-Arv & & & $\mathrm{X}$ & $\mathrm{X}$ & & & \\
\hline Ixora brevifolia Benth. & 41579 & Menezes, M. O. T. & 26 & Farinha-seca & Arb & & & & $\mathrm{X}$ & & & \\
\hline $\begin{array}{l}\text { Margaritopsis carrascoana (Delprete \& E.B.Souza) } \\
\text { C.M.Taylor \& E.B.Souza * }\end{array}$ & 38452 & Castro, A. S. F. & 1607 & & Subarb & & & $\mathrm{X}$ & $\mathrm{x}$ & & & \\
\hline Mitracarpus salzmannianus DC. & 34242 & Vieira, A. V. & sn & & Her & & $\mathrm{x}$ & & $\mathrm{X}$ & $\mathrm{X}$ & & \\
\hline Pentodon pentandrus (Schumach. \& Thonn.) Vatke & 48156 & Castro, A. S. F. & 2383 & & Her & & & & & $\mathrm{X}$ & & \\
\hline Randia armata (Sw.) DC. & 34797 & Silveira, E. & sn & Espinho-de-judeu & Arb & & & $\mathrm{X}$ & $\mathrm{x}$ & & $\mathrm{X}$ & \\
\hline Richardia grandiflora (Cham. \& Schltdl.) Steud. & 33533 & Vieira, A. V. & sn & & Her & & $\mathrm{X}$ & & $\mathrm{X}$ & $\mathrm{X}$ & & \\
\hline $\begin{array}{l}\text { Tocoyena sellowiana (Cham. \& Schltdl.) K.Schum. } \\
\text { RUTACEAE }\end{array}$ & 42572 & Moro, M. F. & 370 & Jenipapo-bravo & Arb & & $\mathrm{X}$ & $\mathrm{X}$ & $\mathrm{x}$ & & & \\
\hline Sigmatanthus trifoliatus Huber ex Emmerich & 31380 & Castro, A. S. F. & 1174 & & Arb & & & & $\mathrm{X}$ & & & \\
\hline Zanthoxylum syncarpum Tul. & 42581 & Moro, M. F. & 359 & Limãozinho & Arv & & & & $\mathrm{x}$ & & & \\
\hline SALICACEAE & & & & & & & & & & & & \\
\hline
\end{tabular}


Tabela 1. Continuação

\begin{tabular}{|c|c|c|c|c|c|c|c|c|c|c|c|}
\hline Espécie & EAC & Coletor & $\mathrm{NC}$ & Nome popular & Hábito & P1 & P2 & $\mathrm{D}$ & $\mathrm{T}$ & A & C M \\
\hline Casearia guianensis (Aubl.) Urb. * & 31371 & Castro, A. S. F. & 1165 & Café-bravo & Arb & & & $\mathrm{X}$ & $\mathrm{X}$ & & \\
\hline Casearia sp. & 49028 & Castro, A. S. F. & 2453 & Cunhão-de-soim & Arb & & & $\mathrm{X}$ & $\mathrm{X}$ & & \\
\hline \multicolumn{12}{|l|}{ SANTALACEAE } \\
\hline Phoradendron sp. & 41466 & Moro, M. F. & 291 & & Hemi-pa & & & & $\mathrm{X}$ & & \\
\hline \multicolumn{12}{|l|}{ SAPINDACEAE } \\
\hline Cardiospermum corindum L. * & 39670 & Castro, A. S. F. & 1757 & & Trep & & & $\mathrm{X}$ & $\mathrm{X}$ & & \\
\hline Talisia esculenta (Cambess.) Radlk. * & 48186 & Castro, A. S. F. & 2413 & Pitomba & Arv & & & & $\mathrm{X}$ & & \\
\hline Serjania obtusidentata Radlk. & 48175 & Castro, A. S. F. & 2402 & & Trep & & & & $\mathrm{X}$ & & \\
\hline \multicolumn{12}{|l|}{ SAPOTACEAE } \\
\hline Chrysophyllum arenarium Allemão & 41498 & Moro, M. F. & 240 & Mamão-de-bode & Arb & & & $\mathrm{X}$ & $\mathrm{X}$ & & \\
\hline Manilkara triflora (Allemão) Monach. & 41495 & Moro, M. F. & 247 & Massaranduba & Arv & & & $\mathrm{X}$ & $\mathrm{X}$ & & \\
\hline Pouteria ramiflora (Mart.) Radlk. & 23440 & Castro, A. S. F. & 43 & Bacumixá & Arv & & & $\mathrm{X}$ & $\mathrm{X}$ & & \\
\hline \multicolumn{12}{|l|}{ SCHOEPFIACEAE } \\
\hline Schoepfia brasiliensis A.DC. & 41580 & Menezes, M. O. T. & 25 & & Arv & & & $\mathrm{X}$ & $\mathrm{X}$ & & \\
\hline \multicolumn{12}{|l|}{ SIMAROUBACEAE } \\
\hline Simaba trichilioides A.St.-Hil. & 41491 & Moro, M. F. & 253 & & Arb & & & & $\mathrm{X}$ & & \\
\hline Simarouba versicolor A.St.-Hil. & 41537 & Moro, M. F. & 276 & Praíba; Paraíba & Arv & & & & $\mathrm{X}$ & & \\
\hline \multicolumn{12}{|l|}{ SOLANACEAE } \\
\hline Cestrum axillare Vell. & 47003 & Ferreira, R. G. & 108 & & Arb & & & $\mathrm{X}$ & $\mathrm{X}$ & & \\
\hline Physalis angulata L. (invasora) & 49030 & Castro, A. S. F. & 2455 & Canapum & Subarb & & & & $\mathrm{X}$ & & \\
\hline Solanum paludosum Moric. & 41503 & Moro, M. F. & 239 & Jurubeba-vermelha & Arb & & & $\mathrm{X}$ & $\mathrm{X}$ & & \\
\hline Solanum paniculatum $\mathrm{L}$. & 33076 & Lima, M. F. & sn & Jurubeba & Arb & & & $\mathrm{X}$ & $\mathrm{X}$ & & \\
\hline \multicolumn{12}{|l|}{ TRIGONIACEAE } \\
\hline Trigonia nivea Cambess. & 42539 & Moro, M. F. & 394 & & Trep & & & $\mathrm{X}$ & $\mathrm{X}$ & & \\
\hline \multicolumn{12}{|l|}{ TURNERACEAE } \\
\hline Piriqueta guianensis N.E.Br. * & 39576 & Castro, A. S. F. & 1710 & & Her & & & & $\mathrm{X}$ & & $\mathrm{X}$ \\
\hline Turnera calyptrocarpa Urb. & 48023 & Castro, A. S. F. & 2374 & & Subarb & & & $\mathrm{X}$ & $\mathrm{X}$ & & \\
\hline Turnera diffusa Willd. ex Schult. & 42549 & Moro, M. F. & 385 & & Subarb & & & & $\mathrm{X}$ & & \\
\hline Turnera subulata Sm. & 34228 & Vieira, A. V. & sn & Chanana & Subarb & & $\mathrm{X}$ & & $\mathrm{X}$ & & \\
\hline \multicolumn{12}{|l|}{ URTICACEAE } \\
\hline Cecropia palmata Willd. & 44645 & Ferreira, R. G. & 56 & Torém & Arv & & & $\mathrm{X}$ & $\mathrm{X}$ & & \\
\hline \multicolumn{12}{|l|}{ VERBENACEAE } \\
\hline Lantana camara L. & 47399 & Castro, A. S. F. & 2340 & Chumbinho & Arb & & $\mathrm{X}$ & $\mathrm{X}$ & $\mathrm{X}$ & $\mathrm{X}$ & $\mathrm{X}$ \\
\hline Lantana fucata Lindl. & 49085 & Castro, A. S. F. & 2471 & & Arb & & & $\mathrm{X}$ & $\mathrm{X}$ & & \\
\hline Stachytarpheta sessilis Moldenke & 49083 & Castro, A. S. F. & 2469 & Pescoço-de-ganso & Her & & & & $\mathrm{X}$ & $\mathrm{X}$ & \\
\hline \multicolumn{12}{|l|}{ VIOLACEAE } \\
\hline Hybanthus calceolaria (L.) Oken & 41568 & Menezes, M. O. T. & 6 & Pepaconha & Her & & $\mathrm{X}$ & & $\mathrm{X}$ & $\mathrm{X}$ & \\
\hline \multicolumn{12}{|l|}{ VITACEAE } \\
\hline Cissus erosa Rich. & 49090 & Castro, A. S. F. & 2476 & & Trep & & & & $\mathrm{X}$ & & \\
\hline Cissus tinctoria Mart. & 50033 & Magalhães, H. & 189 & & Trep & & & $\mathrm{X}$ & & & \\
\hline Cissus verticillata (L.) Nicolson \& C.E Jarvis & 49133 & Castro, A. S. F. & 2490 & Insulina & Trep & & & $\mathrm{X}$ & $\mathrm{X}$ & & \\
\hline \multicolumn{12}{|l|}{ XYRIDACEAE } \\
\hline Xyris laxifolia Mart. & 41521 & Moro, M. F. & 334 & & Her & & & & & $\mathrm{X}$ & \\
\hline \multicolumn{12}{|l|}{ INDETERMINADA } \\
\hline Indeterminada & & Observada & & & Trep & & & & $\mathrm{X}$ & & \\
\hline
\end{tabular}

de 3,8 m e desvio padrão de $1,03 \mathrm{~m}$, com alguns indivíduos arbóreos atingindo $8 \mathrm{~m}$ de altura, mas com $82 \%$ deles menores que $4 \mathrm{~m}$ (Fig. 4). A dominância total da comunidade (áreas basais de todos os indivíduos vivos somadas) foi de $39,28 \mathrm{~m}^{2} /$ ha.

Mesmo possuindo um regime pluviométrico tropical seco subúmido (com déficit hídrico e com forte estacionalidade), devido à influência da umidade oceânica a área estudada pode apresentar precipitações médias anuais até duas vezes maiores que em áreas continentais da depressão sertaneja e períodos anuais de estiagem não maiores que 6 meses (Nimer, 1972). Além disso, a região diferencia-se de áreas continentais pela influência da maresia (salinidade), dos solos profundos e lixiviados, bem como pela maior pluviosidade. Deste modo, é esperado que a composição florística das vegetações litorâneas seja diferente daquela registrada na Caatinga. Este estudo, por exemplo, registrou espécies de diferentes domínios fitogeográficos na área levantada, ressaltando a mistura de espécies característica das formações do LSN. 
Tabela 2. Fitossociologia da mata de tabuleiro amostrada no Pecém, Ceará. AB - abundância; DA - densidade absoluta (plantas/ha); DR - densidade relativa; FA frequência absoluta; FR - frequência relativa; DoA - dominância absoluta ( $\left.\mathrm{m}^{2} / \mathrm{ha}\right)$; DoR - dominância relativa; IVI - Índice de Valor de Importância (DR + FR + DoR).

\begin{tabular}{|c|c|c|c|c|c|c|c|c|}
\hline Espécies & $\mathrm{AB}$ & DA & $\mathrm{DR}$ & FA & FR & DoA & DoR & IVI \\
\hline Chamaecrista ensiformis (Fabaceae) & 417 & 1303,1 & 14 & 100 & 4,23 & 11,07 & 28,2 & 46,5 \\
\hline Manilkara triflora (Sapotaceae) & 421 & 1315,6 & 14,2 & 100 & 4,23 & 5,16 & 13,1 & 31,5 \\
\hline Guapira nitida (Nyctaginaceae) & 297 & 928,1 & 10 & 100 & 4,23 & 4,24 & 10,8 & 25 \\
\hline Cordiera sessilis (Rubiaceae) & 339 & 1059,4 & 11,4 & 100 & 4,23 & 1,4 & 3,56 & 19,2 \\
\hline Maytenus erythroxyla (Celastraceae) & 273 & 853,1 & 9,19 & 100 & 4,23 & 1,27 & 3,24 & 16,7 \\
\hline Byrsonima gardneriana (Malpighiaceae) & 137 & 428,1 & 4,61 & 100 & 4,23 & 3,06 & 7,8 & 16,6 \\
\hline Mouriri cearensis (Melastomataceae) & 151 & 471,9 & 5,08 & 87,5 & 3,7 & 2,73 & 6,94 & 15,7 \\
\hline Psidium sartorianum (Myrtaceae) & 171 & 534,4 & 5,76 & 100 & 4,23 & 0,88 & 2,25 & 12,2 \\
\hline Ouratea sp. (Ochnaceae) & 126 & 393,8 & 4,24 & 87,5 & 3,7 & 1,29 & 3,3 & 11,2 \\
\hline Strychnos parvifolia (Loganiaceae) & 76 & 237,5 & 2,56 & 100 & 4,23 & 0,42 & 1,07 & 7,86 \\
\hline Erythroxylum barbatum (Erythroxylaceae) & 64 & 200 & 2,15 & 100 & 4,23 & 0,43 & 1,09 & 7,48 \\
\hline Coccoloba ramosissima (Polygonaceae) & 67 & 209,4 & 2,26 & 87,5 & 3,7 & 0,31 & 0,79 & 6,75 \\
\hline Protium heptaphyllum (Burseraceae) & 50 & 156,3 & 1,68 & 50 & 2,12 & 1,15 & 2,92 & 6,72 \\
\hline Myrcia guianensis (Myrtaceae) & 36 & 112,5 & 1,21 & 75 & 3,17 & 0,69 & 1,75 & 6,13 \\
\hline Guettarda angelica (Rubiaceae) & 32 & 100 & 1,08 & 75 & 3,17 & 0,19 & 0,49 & 4,74 \\
\hline Handroanthus impetiginosus (Bignoniaceae) & 35 & 109,4 & 1,18 & 25 & 1,06 & 0,96 & 2,43 & 4,67 \\
\hline Parkia platycephala (Fabaceae) & 7 & 21,9 & 0,24 & 37,5 & 1,59 & 1 & 2,56 & 4,38 \\
\hline Tabebuia roseoalba (Bignoniaceae) & 19 & 59,4 & 0,64 & 50 & 2,12 & 0,38 & 0,97 & 3,73 \\
\hline Ipomoea subincana (Convolvulaceae) & 14 & 43,8 & 0,47 & 62,5 & 2,65 & 0,2 & 0,51 & 3,62 \\
\hline Agonandra brasiliensis (Opiliaceae) & 17 & 53,1 & 0,57 & 50 & 2,12 & 0,23 & 0,58 & 3,27 \\
\hline Tocoyena sellowiana (Rubiaceae) & 7 & 21,9 & 0,24 & 62,5 & 2,65 & 0,03 & 0,08 & 2,96 \\
\hline Plathymenia reticulata (Fabaceae) & 6 & 18,8 & 0,2 & 37,5 & 1,59 & 0,4 & 1,01 & 2,8 \\
\hline Myrcia splendens (Myrtaceae) & 12 & 37,5 & 0,4 & 50 & 2,12 & 0,1 & 0,25 & 2,77 \\
\hline Mansoa sp. (Bignoniaceae) & 31 & 96,9 & 1,04 & 25 & 1,06 & 0,18 & 0,46 & 2,56 \\
\hline Ocotea canaliculata (Lauraceae) & 7 & 21,9 & 0,24 & 50 & 2,12 & 0,07 & 0,19 & 2,54 \\
\hline Calliandra sessilis (Fabaceae) & 33 & 103,1 & 1,11 & 25 & 1,06 & 0,1 & 0,25 & 2,42 \\
\hline Himatanthus drasticus (Apocynaceae) & 7 & 21,9 & 0,24 & 37,5 & 1,59 & 0,22 & 0,56 & 2,38 \\
\hline Davilla cearensis (Dilleniaceae ) & 5 & 15,6 & 0,17 & 50 & 2,12 & 0,02 & 0,05 & 2,33 \\
\hline Manihot sp. (Euphorbiaceae) & 15 & 46,9 & 0,51 & 37,5 & 1,59 & 0,05 & 0,13 & 2,23 \\
\hline Duguetia riedeliana (Annonaceae) & 27 & 84,4 & 0,91 & 12,5 & 0,53 & 0,28 & 0,71 & 2,15 \\
\hline Schoepfia brasiliensis (Schoepfiaceae) & 8 & 25 & 0,27 & 37,5 & 1,59 & 0,09 & 0,22 & 2,08 \\
\hline Pilosocereus catingicola subsp. salvadorensis (Cactaceae) & 5 & 15,6 & 0,17 & 37,5 & 1,59 & 0,07 & 0,18 & 1,94 \\
\hline Tetracera willdenowiana (Dilleniaceae) & 18 & 56,3 & 0,61 & 25 & 1,06 & 0,11 & 0,27 & 1,94 \\
\hline Hirtella ciliata (Chrysobalanaceae) & 5 & 15,6 & 0,17 & 25 & 1,06 & 0,11 & 0,27 & 1,5 \\
\hline Byrsonima crassifolia (Malpighiaceae) & 6 & 18,8 & 0,2 & 25 & 1,06 & 0,06 & 0,15 & 1,42 \\
\hline Erythroxylum laetevirens (Erythroxylaceae) & 3 & 9,4 & 0,1 & 25 & 1,06 & 0,01 & 0,02 & 1,18 \\
\hline Chiococca alba (Rubiaceae) & 3 & 9,4 & 0,1 & 25 & 1,06 & 0,01 & 0,02 & 1,18 \\
\hline Chloroleucon acacioides (Fabaceae) & 1 & 3,1 & 0,03 & 12,5 & 0,53 & 0,07 & 0,18 & 0,74 \\
\hline Anacardium occidentale (Anacardiaceae) & 1 & 3,1 & 0,03 & 12,5 & 0,53 & 0,07 & 0,18 & 0,74 \\
\hline Parinari campestris (Chrysobalanaceae) & 3 & 9,4 & 0,1 & 12,5 & 0,53 & 0,04 & 0,1 & 0,73 \\
\hline Myrtaceae indeterminada & 3 & 9,4 & 0,1 & 12,5 & 0,53 & 0,02 & 0,05 & 0,68 \\
\hline Leptolobium dasycarpum (Fabaceae) & 1 & 3,1 & 0,03 & 12,5 & 0,53 & 0,04 & 0,11 & 0,67 \\
\hline Sterculia striata (Malvaceae) & 1 & 3,1 & 0,03 & 12,5 & 0,53 & 0,03 & 0,08 & 0,64 \\
\hline Trepadeira indeterminada & 2 & 6,3 & 0,07 & 12,5 & 0,53 & 0,01 & 0,02 & 0,62 \\
\hline Faramea nitida (Rubiaceae) & 2 & 6,3 & 0,07 & 12,5 & 0,53 & 0,01 & 0,02 & 0,62 \\
\hline Coccoloba latifolia (Polygonaceae) & 2 & 6,3 & 0,07 & 12,5 & 0,53 & $<0,01$ & 0,01 & 0,61 \\
\hline Eugenia luschnathiana (Myrtaceae ) & 2 & 6,3 & 0,07 & 12,5 & 0,53 & $<0,01$ & 0,01 & 0,61 \\
\hline Serjania obtusidentata (Sapindaceae) & 1 & 3,1 & 0,03 & 12,5 & 0,53 & 0,01 & 0,03 & 0,59 \\
\hline Campomanesia aromatica (Myrtaceae) & 1 & 3,1 & 0,03 & 12,5 & 0,53 & 0,01 & 0,02 & 0,58 \\
\hline Eugenia sp.2 (Ubaitinga - Myrtaceae) & 1 & 3,1 & 0,03 & 12,5 & 0,53 & $<0,01$ & 0,01 & 0,57 \\
\hline Myrcia sp. (Mapirunga - Myrtaceae) & 1 & 3,1 & 0,03 & 12,5 & 0,53 & $<0,01$ & 0,01 & 0,57 \\
\hline Buchenavia tetraphylla (Combretaceae) & 1 & 3,1 & 0,03 & 12,5 & 0,53 & $<0,01$ & 0,01 & 0,57 \\
\hline Mortas & 118 & 368,8 & - & - & - & 1,19 & - & - \\
\hline Total & 2970 & 9281,7 & 100,0 & 2362,5 & 100,0 & 39,28 & 100,0 & 300,0 \\
\hline
\end{tabular}




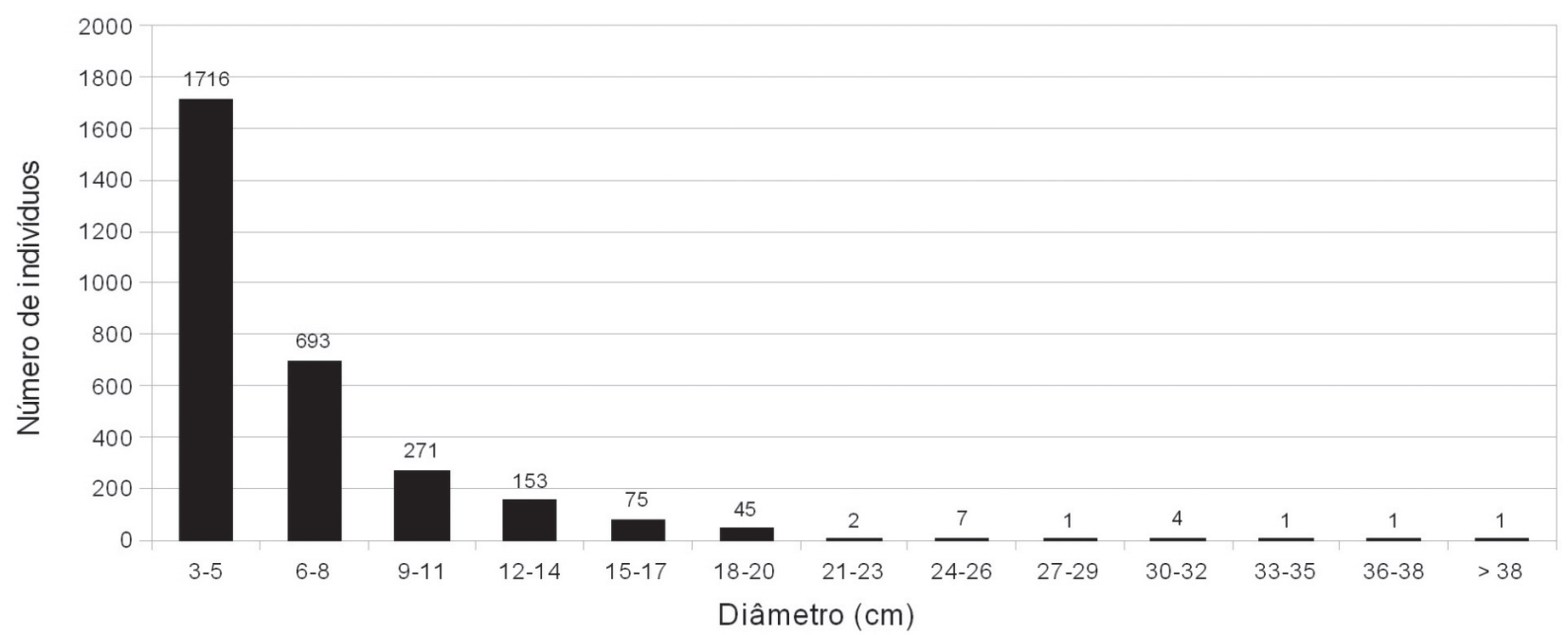

Figura 3. Classes de diâmetro da comunidade lenhosa da "mata de tabuleiro" amostrada no Pecém, Ceará.

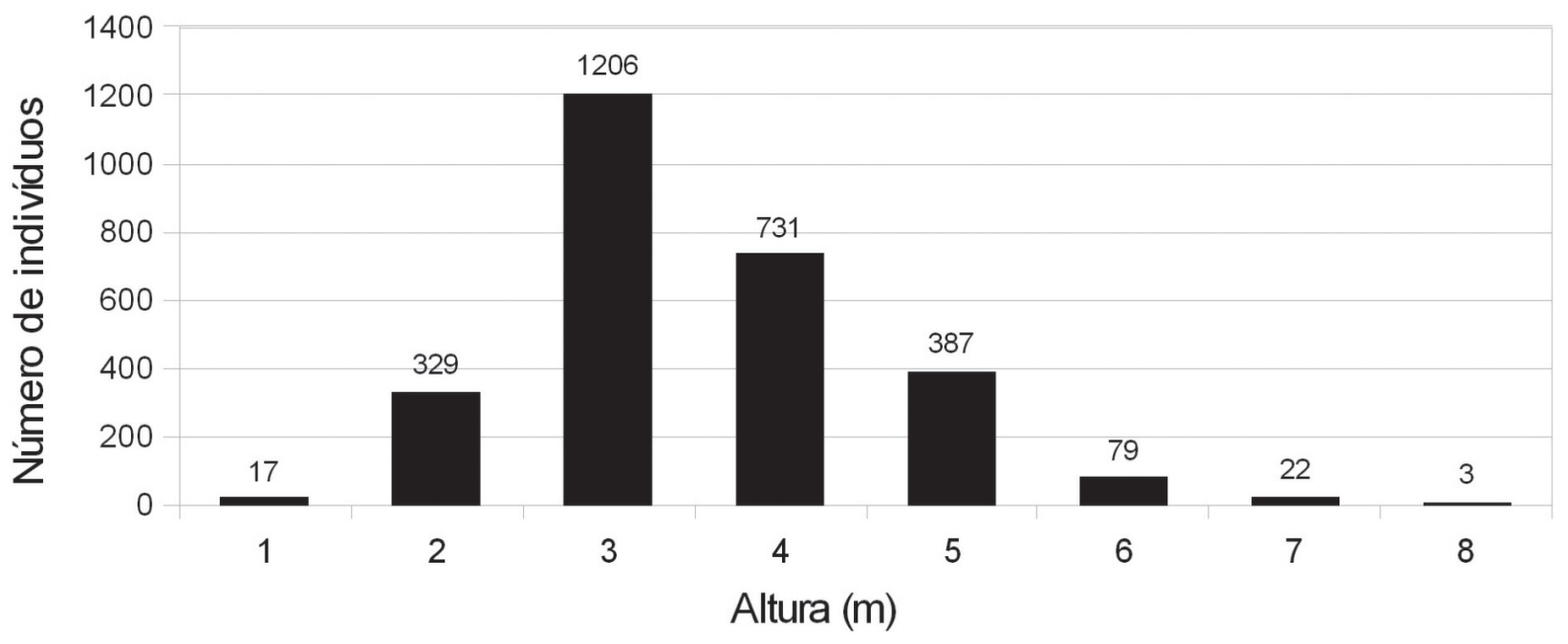

Figura 4. Classes de altura da comunidade lenhosa da "mata de tabuleiro" amostrada no Pecém, Ceará.

A mistura de espécies no litoral cearense torna particularmente difícil a classificação de suas formações. Enquanto Brasil (2004) posiciona a costa setentrional no Domínio das Caatingas, Fernandes (1998) considera toda a costa brasileira como pertencente à Sub-província Litorânea da Província Atlântica. Já Ab’Sáber (2003), em seu mapa dos Domínios Morfoclimáticos Brasileiros, considera parte da costa cearense como "Faixa de transição - não diferenciadas", o que chama a atenção para as condições intermediárias do local estudado. A flora local é constituída por um misto de espécies de diferentes domínios, o que já chamava a atenção de autores anteriores (Fernandes 1990; 1998; Figueiredo 1997). Rizzini (1963, p. 32), por exemplo, pontua que "os tabuleiros nordestinos assentam sobre estreita faixa da Formação Barreiras (Terciário) e levam flora mista (cerrado plus restinga), ao passo que a restinga oriental [...] é de origem atlântica pura". Embora considere o LSN per- tencente à Província Atlântica, Fernandes (1998, p. 248) também reconhece que "o tabuleiro, mais comumente usado no Norte/Nordeste, é marcado por um complexo florístico, dada a co-participação de elementos da vegetação vizinha: mata, caatinga e formações esclerófilas - cerrado e cerradão".

Werneck et al. (2011), modelando a distribuição da Caatinga durante as glaciações pleistocênicas, mostraram que algumas áreas de Mata Atlântica do leste nordestino (Pernambuco e Bahia), bem como parte da costa cearense, não foram passíveis de ocupação pela Caatinga nem mesmo nos períodos mais secos do Pleistoceno (Werneck et al. 2011, Fig. 4, p. 279). Os modelos não previram a ocorrência de Caatinga durante o último máximo glacial no trecho da costa estudado por esse trabalho, sugerindo que as diferenças climáticas entre a costa e as caatingas vem sendo impostas à biota por um tempo relativamente longo, o que reforça a distinção das formações costeiras (vegetação 
pioneira psamófila, floresta de dunas fixas e vegetação dos tabuleiros) da Caatinga.

Embora algumas espécies da Caatinga tenham sucesso ao explorar os ambientes costeiros, a flora como um todo tende a ser um misto de espécies de diferentes domínios fitogeográficos, que aproveitam as condições climáticas intermediárias para se estabelecer. Assim, a costa setentrional do Nordeste brasileiro parece servir, em maior ou menor grau, como um corredor ecológico entre o Cerrado de um lado e a Mata Atlântica do outro, bordejado pela Caatinga. Esse mosaico de condições ambientais permite a coexistência de espécies de caatinga, de cerrado, espécies psamófilas e até espécies florestais. Espécies de ampla ocorrência no domínio dos Cerrados (e.g. Byrsonima crassifolia, Stryphnodendron coriaceum, Curatella americana, Anacardium occidentale, Leptolobium dasycarpum e Annona coriacea), por exemplo, são plantas comuns na região costeira do Ceará (Moro et al. 2011, Tabela 1). Mas além de elementos do Cerrado há também a presença de espécies características do Domínio das Caatingas, a exemplo de Cereus jamacaru, Pityrocarpa moniliformis, Luetzelburgia auriculata e Croton blanchetianus. Por fim, temos a ocorrência de espécies de ampla distribuição, que ocorrem em diversos biomas (e.g. Ximenia americana, Tapirira guianensis, Handroanthus impetiginosus), e mesmo espécies mais características da Amazônia (e.g. Coccoloba latifolia, Tetracera willdenowiana), que se somam às plantas típicas das restingas (e.g. Ipomoea pes-caprae, Remirea maritima, Eugenia luschnathiana e Chrysobalanus icaco) para constituir a flora do LSN.

Em uma meta-análise sobre os vínculos florísticos dos "brejos de altitude" da Paraíba, Pernambuco e Sergipe com a caatinga (áreas secas) e a mata atlântica (áreas úmidas), Rodal et al. (2008) encontraram gêneros mais afeitos a locais mais úmidos e outros a locais mais secos. O litoral cearense possui solos mais profundos por sua natureza sedimentar do que no embasamento cristalino e pluviosidade em geral maior que em áreas de caatinga do interior do Ceará, possibilitando uma maior disponibilidade hídrica. Assim, encontramos tanto táxons indicados por Rodal et al. (2008) como de áreas preferencialmente úmidas quanto de áreas preferencialmente secas.

Chamaecrista, Manilkara, Tapirira, Annona, Xylopia, Himatanthus, Tabernaemontana, Protium, Cecropia, Maytenus, Hirtella, Clusia, Buchenavia e Ficus são exemplos de gêneros amostrados neste estudo que, segundo Rodal et al. (2008), são mais afeitos a áreas mais úmidas. Entretanto, a presença de Cereus, Pilosocereus, Croton, Ziziphus, Guettarda, Chloroleucon e vários outros gêneros sugeridos como mais afeitos a áreas secas mostram o caráter intermediário do local. Se o clima não é tão úmido quanto em áreas de floresta atlântica, também não é tão seco quanto em áreas de Caatinga, possibilitando uma mistura considerável de táxons.

Em relação à estrutura da vegetação, a amostragem fitossociológica mostrou uma densidade de indivíduos bastante alta (9.281,25 ind/ha), especialmente quando com- paramos a vegetação estudada com a de áreas de caatinga (e.g. Alcoforado-Filho et al. 2003 - $3.810 \mathrm{ind} / \mathrm{ha}$; Amorim et al. 2005 - $3.247 \mathrm{ind} / \mathrm{ha}$; Lemos \& Rodal $2002-5.827$ ind/ha; Calixto Júnior \& Drumond $2011-1.350$ ind/ha) e de cerrado costeiro (Moro et al. $2011-1.218 \mathrm{ind} / \mathrm{ha}$ ). De fato, a maior disponibilidade hídrica e os solos profundos observados na zona costeira parecem possibilitar uma maior densidade de indivíduos que na caatinga. No entanto, o alto valor da densidade em parte pode provavelmente ser atribuído à grande proporção de indivíduos de pequeno porte (Fig. 3), já que a área amostrada é uma floresta secundária. O corte seletivo de Chamaecrista ensiformis e Manilkara triflora também induz ramificações excessivas nessas plantas (muitas vezes abaixo do solo), resultando em uma densidade inflada.

As matas de tabuleiro são estrutural e fisionomicamente muito distintas do cerrado costeiro estudado por Moro et al. (2011), pois formam um dossel e a densidade e área basal são bem superiores mas ambas as formações possuem elementos florísticos em comum (e.g. Anacardium occidentale; Mouriri cearensis; Himatanthus drasticus; Byrsonima crassifolia etc.), ressaltando o fato de que as formações do LSN são compostas por espécies de diversos domínios fitogeográficos que ocuparam a Formação Barreiras e a planície litorânea.

As florestas de tabuleiro são conhecidas por terem porte baixo, mas a altura média dos indivíduos na área estudada parece estar abaixo do potencial que a comunidade pode atingir. Observamos fora das parcelas exemplares grandes de Parkia platycephala e Anacardium occidentale com até 6-9(10) m de altura e diâmetro de $30-50 \mathrm{~cm}$, e amostramos algumas poucas árvores nas parcelas com até $8 \mathrm{~m}$ de altura, o que parece ser o porte de várias espécies de mata de tabuleiro na maturidade.

\section{Conclusão}

Existem ainda poucos estudos florísticos e fitossociológicos que contemplem comunidades vegetais do complexo vegetacional litorâneo do LSN, mas sugerimos que as peculiaridades climáticas, geológicas e pedológicas na área estudada a tornam uma região ecotonal, por incluir elementos florísticos de diferentes domínios fitogeográficos brasileiros. Contudo, apesar da singularidade da vegetação do LSN, não foram registradas espécies endêmicas neste estudo, o que é esperado, devido à idade relativamente recente da Formação Barreiras e dos campos de dunas geoambientes dominantes no litoral setentrional.

No que diz respeito à conservação do complexo vegetacional do litoral setentrional, tem se observado grande conflito entre as atividades degradadoras e as iniciativas de conservação. Por um lado, os ecossistemas naturais da região sofrem grande pressão devido a empreendimentos turísticos, industriais e agrícolas; por outro lado, a região costeira tem sido prioridade para instalação de unidades de conservação no Ceará (Menezes et al. 2011). No caso específico do 
Pecém, a maior fonte de impactos é o Complexo Portuário e Industrial do Pecém, que poderá eliminar ou substituir em poucas décadas grande parte da cobertura vegetal em uma área onde existem apenas três áreas protegidas (APA do Pecém - 122,79 ha; Estação Ecológica do Pecém - 973 ha; e o Jardim Botânico de São Gonçalo do Amarante - 19 ha), de extensão limitada.

A condição de ecótono do LSN lhe proporciona grande potencial para o teste de hipóteses ecológicas, principalmente no que diz respeito à fitogeografia e à competição entre espécies vegetais. Levantamentos florísticos e fitossociológicos em outros setores do LSN são fortemente desejáveis, no intuito de aprimorar o conhecimento sobre a florística e a estrutura de suas comunidades vegetais. Estudos de sensoriamento remoto também são desejáveis, no intuito de melhor delimitar a distribuição do Complexo Vegetacional Litorâneo e detectar sítios prioritários para conservação biológica.

\section{Agradecimentos}

Os autores agradecem a Adalberto Maciel Mano de Carvalho, Sarah Sued Gomes de Souza e Regina Celli Araújo de Freitas, do herbário EAC, pelo auxílio durante as consultas e depósito de material junto ao herbário; M. F. Moro agradece à Coordenação de Aperfeiçoamento de Pessoal de Nível Superior (CAPES) e à Fundação de Amparo à Pesquisa do Estado de São Paulo (FAPESP) pelas bolsas de pós-graduação concedidas.

\section{Referências}

Ab’Sáber, A.N. 2001. Litoral do Brasil. São Paulo, Metalivros.

Ab'Sáber, A.N. 2003. Os domínios de natureza no Brasil: potencialidades paisagísticas. São Paulo, Ateliê Editorial.

Ab'Sáber, A.N. 2006. Brasil: paisagens de exceção: o litoral e o pantanal mato-grossense: patrimônios básicos. Cotia, Ateliê Editorial.

Alcoforado-Filho, F.G.; Sampaio, E.V.D.S.B. \& Rodal, M.J.N. 2003. Florística e fitossociologia de um remanescente de vegetação caducifólia espinhosa arbórea em Caruaru, Pernambuco. Acta Botanica Brasilica 17: 287-303.

Amorim, I.L.D.; Sampaio, E.V.S.B. \& Araújo, E.D.L. 2005. Flora e estrutura da vegetação arbustivo-arbórea de uma área de caatinga do Seridó, RN, Brasil. Acta Botanica Brasilica 19: 615-623.

Andrade-Lima, D. 1981. The caatingas dominium. Revista Brasileira de Botânica 4: 149-153.

APG III. 2009. An update of the Angiosperm Phylogeny Group classification for the orders and families of flowering plants: APG III. Botanical Journal of the Linnean Society 161: 105-121.

Brasil, Instituto Brasileiro de Geografia e Estatística. 2002. Mapa de clima do Brasil. Escala 1:5.000.000. Rio de Janeiro, IBGE
Brasil, Instituto Brasileiro de Geografia e Estatística. 2004. Mapa de biomas do Brasil: primeira aproximação. Escala 1:5.000.000. Rio de Janeiro, IBGE.

Calixto Júnior, J.T. \& Drumond, M.A. 2011. Estrutura fitossociológica de um fragmento de Caatinga sensu stricto 30 anos após corte raso, Petrolina-PE, Brasil. Revista Caatinga 24: 67-74.

Campos, A. A.; Monteiro, A. Q.; Monteiro-Neto, C.; Polette, M. 2003. Zona Costeira do Ceará: Diagnóstico para a gestão integrada. Fortaleza, Aquasis.

Ceará, Instituto de Pesquisa e Estratégia Econômica do Ceará. 2010. Perfil Básico Municipal: São Gonçalo do Amarante. http://www.ipece. ce.gov.br/publicacoes/perfil_basico/pbm-2010/Sao_Goncalo_do_ Amarante.pdf (Acesso em 13/06/2011).

Durigan, G. 2003. Métodos para análise de vegetação arbórea. Pp. 455478 In: Cullen Júnior, L. Rudran, R. \& Valladares-Padua, C. (Eds.). Métodos de estudos em biologia da conservação e manejo da vida silvestre. (Eds). Curitiba. UFPR / Fundação O Boticário de Proteção à Natureza.

Fernandes, A. 1990. Conjunto vegetacional cearense. Pp. 51-98. In: A. Fernandes (Ed.). Temas fitogeográficos. Fortaleza, Stylus Comunicações.

Fernandes, A. 1998. Fitogeografia Brasileira. Fortaleza, Multigraf

Figueiredo, M.A. 1997. A cobertura vegetal do Ceará (Unidades Fitoecológicas) In: Ceará (Ed.). Atlas do Ceará. Fortaleza, IPLANCE.

Filgueiras, T.S.; Nogueira, P.E.; Brochado, A.L. \& Guala II, G.F. 1994 Caminhamento: um método expedito para levantamentos florísticos qualitativos. Cadernos de Geociências 12: 39-43.

Lemos, J.R. \& Rodal, M.J.N. 2002. Fitossociologia do componente lenhoso de um trecho da vegetação de caatinga no Parque Nacional Serra da Capivara, Piauí, Brasil. Acta Botanica Brasilica 16: 23-42.

Matias, L.Q. \& Nunes, E.P. 2001. Levantamento florístico da Área de Proteção Ambiental de Jericoacoara, Ceará. Acta Botanica Brasilica 15: $35-43$.

Menezes, M.O.T.; Araújo, F.S.; Romero, R.E. 2010. O sistema de conservação biológica do estado do Ceará: Diagnóstico e Recomendações. REDE - Revista Eletrônica do Prodema 5(2): 7-31.

Moro, M.F.; Castro, A.S.F. \& Araújo, F.S. 2011. Composição florística e estrutura de um fragmento de vegetação savânica sobre os tabuleiros pré-litorâneos na zona urbana de Fortaleza, Ceará. Rodriguésia 62: 407-423.

Nimer, E. 1972. Climatologia da Região Nordeste do Brasil: subsídios à geografia regional do Brasil, Revista Brasileira de Geografia 34(2): 5-51.

Richardson, D.M.; Pysek, P.; Rejmanek, M.; Barbour, M.G.; Panetta, F.D. \& West, C.J. 2000. Naturalization and Invasion of Alien Plants: Concepts and Definitions. Diversity and Distributions 6: 93-107.

Rizzini, C.T. 1963. Nota prévia sôbre a divisão fitogeográfica (florísticosociológica) do Brasil. Revista Brasileira de Geografia 25(1): 3-64.

Rodal, M.J.N.; Barbosa, M.R.V. \& Thomas, W.W. 2008. Do the seasonal forests in northeastern Brazil represent a single floristic unit? Brazilian Journal of Biology 68: 467-475.

Veloso, H.P.; Rangel-Filho, A.L.R; Lima, J.C.A. 1991. Classificação da vegetação brasileira, adaptada a um sistema universal, Rio de Janeiro, IBGE.

Velloso, A.L.; Sampaio, E.V.S.B. \& Pareyn, F.G.C. 2002. Ecorregiões propostas para o bioma caatinga. Recife, Associação Plantas do Nordeste / The Nature Conservancy do Brasil.

Werneck, F.P.; Costa, G.C.; Colli, G.R.; Prado, D.E. \& Sites Jr, J.W. 2011. Revisiting the historical distribution of seasonally dry tropical forests: new insights based on palaeodistribution modelling and palynological evidence. Global Ecology and Biogeography 20: 272-288. 